NOTICE: this is the author's version of a work that was accepted for publication in Systems and Control Letters. Changes resulting from the publishing process, such as peer review, editing, corrections, structural formatting, and other quality control mechanisms may not be reflected in this document. Changes may have been made to this work since it was submitted for publication. A definitive version was subsequently published in Systems and Control Letters, Vol. 75 (2015). DOI: 10.1016/j.sysconle.2014.11.003 


\title{
Global Inverse Optimal Stabilization of Stochastic Nonholonomic Systems
}

\author{
K.D. Do
}

\begin{abstract}
Optimality has not been addressed in existing works on control of (stochastic) nonholonomic systems. This paper presents a design of optimal controllers with respect to a meaningful cost function to globally asymptotically stabilize (in probability) nonholonomic systems affine in stochastic disturbances. The design is based on the Lyapunov direct method, the backstepping technique, and the inverse optimal control design. A class of Lyapunov functions, which are not required to be as nonlinearly strong as quadratic or quartic, is proposed for the control design. Thus, these Lyapunov functions can be applied to design of controllers for underactuated (stochastic) mechanical systems, which are usually required Lyapunov functions of a nonlinearly weak form. The proposed control design is illustrated on a kinematic cart, of which wheel velocities are perturbed by stochastic noise.
\end{abstract}

\section{Index Terms}

Stochastic nonholonomic systems, global stabilization, inverse optimal.

\section{INTRODUCTION}

This paper presents a design of optimal controllers with respect to a meaningful cost function for global asymptotic stabilization in probability of the following stochastic nonholonomic system

$$
\begin{aligned}
& d x_{0}=u_{0} d t+\boldsymbol{\varphi}_{0}^{T}\left(x_{0}\right) d \boldsymbol{w}, \\
& d x_{i}=x_{i+1} u_{0} d t+\boldsymbol{\varphi}_{i}^{T}\left(x_{0}, u_{0}, \overline{\boldsymbol{x}}_{i}\right) d \boldsymbol{w}, \quad 1 \leq i \leq n-1 \\
& d x_{n}=u_{1} d t+\boldsymbol{\varphi}_{n}^{T}\left(x_{0}, u_{0}, \boldsymbol{x}\right) d \boldsymbol{w},
\end{aligned}
$$

where $u_{0}$ and $u_{1}$ are controls, $x_{0}$ and $\boldsymbol{x}=\operatorname{col}\left(x_{1}, \ldots, x_{n}\right)$ are system states, $\overline{\boldsymbol{x}}_{i}=\operatorname{col}\left(x_{1}, \ldots, x_{i}\right), \boldsymbol{w}$ is an independent $r$-dimensional standard Wiener process, and $\varphi_{0}(\bullet)$ and $\varphi_{i}(\bullet)$ are $r$-vector valued smooth functions satisfying the following assumption:

Assumption 1.1: The vector valued smooth functions $\boldsymbol{\varphi}_{0}\left(x_{0}\right), \boldsymbol{\varphi}_{i}\left(x_{0}, u_{0}, \overline{\boldsymbol{x}}_{i}\right)$, and $\boldsymbol{\varphi}_{n}\left(x_{0}, u_{0}, \boldsymbol{x}\right)$ vanish at the origin.

The above assumption implies that the origin is the equilibrium point of the system (1) and is imposed to guarantee controllability of the $\boldsymbol{x}$-subsystem, i.e., the last two equations of (1), in the limit when $x_{0} \rightarrow 0$ as $t \rightarrow \infty$. For clarity, the system (1) does not include nonlinear deterministic functions and unknown noise covariance. Including these terms does not add contributions but increases complexity of presentation because if there are deterministic functions (either containing unknown parameters or not) and/or unknown noise covariance, it is rather straightforward to combine the control design proposed in this paper together with techniques in [1] and [2] to deal with these functions containing both linear and nonlinear appearance of unknown parameters and noise covariance.

Let us consider the following kinematic cart that motivates the study of the stochastic nonholonomic system (1).

Example 1.1: The kinematic cart, see Fig. 1, is described by [3]:

$$
\left[\begin{array}{c}
\dot{x} \\
\dot{y} \\
\dot{\phi}
\end{array}\right]=\frac{s}{4}\left[\begin{array}{cc}
\cos (\phi) & \cos (\phi) \\
\sin (\phi) & \sin (\phi) \\
\frac{2}{b} & -\frac{2}{b}
\end{array}\right]\left[\begin{array}{l}
\nu_{1} \\
\nu_{2}
\end{array}\right]
$$

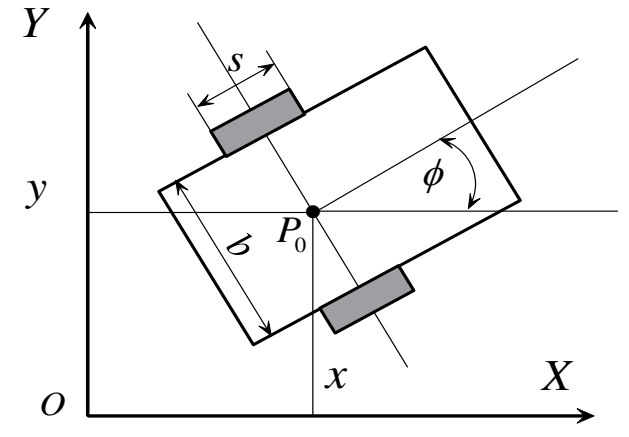

Fig. 1: Cart parameters and coordinates 
where $(x, y)$ and $\phi$ denote the position and orientation of the cart, $s$ is the diameter of the actuated wheel, $b$ is the width, $P_{0}$ is the middle point between the two actuated wheels, and $\nu_{1}$ and $\nu_{2}$ are the angular velocities of the actuated wheels. We now suppose that the angular velocities $\nu_{1}$ and $\nu_{2}$ are subject to some stochastic disturbances, and are assumed to be expressed as [4]:

$$
\nu_{i}=\bar{\nu}_{i}(x, y, \phi)+\zeta_{0}(x, y, \phi) \dot{w}, i=1,2,
$$

where $\bar{\nu}_{i}(x, y, \phi), i=1,2$ are viewed as controls, $\zeta_{0}(x, y, \phi)$ is a function of $(x, y, \phi)$ and vanishes at the origin, and $w$ is a standard Wiener process. The following coordinate changes

$$
\begin{aligned}
& {\left[\begin{array}{l}
x_{0} \\
x_{1} \\
x_{2}
\end{array}\right]=\left[\begin{array}{ccc}
0 & 0 & 1 \\
\sin (\phi) & -\cos (\phi) & 0 \\
\cos (\phi) & \sin (\phi) & 0
\end{array}\right]\left[\begin{array}{l}
x \\
y \\
\phi
\end{array}\right],} \\
& u_{0}=\frac{s}{2 b}\left(\bar{\nu}_{1}-\bar{\nu}_{2}\right), u_{1}=\frac{s}{4}\left(\bar{\nu}_{1}+\bar{\nu}_{2}\right)+x_{1} u_{0}
\end{aligned}
$$

transform the kinematic cart (2) together with (3) to

$$
\begin{aligned}
& d x_{0}=u_{0} d t \\
& d x_{1}=u_{0} x_{2} d t \\
& d x_{2}=u_{1} d t+\frac{s}{2} \zeta_{0}\left(x_{0}, x_{1}, x_{2}\right) d w
\end{aligned}
$$

which is a special form of the stochastic nonholonomic system (1). We will continue this example in Subsections III-C, IV-B, and V-C.

When $d \boldsymbol{w} / d t$ is an either known or unknown constant vector, the system (1) becomes deterministic. By Brockett's condition [5], deterministic nonholonomic systems cannot be stabilized at the origin by any static continuous state feedback though they are open loop controllable. To overcome this obstacle, researchers have developed novel approaches to design asymptotic/exponential stabilizers, see for example [6], [7], [8], [9], [10], [1], [11] on the discontinuous time-invariant approach, and [12], [13], [14], [15] on the time-varying approach.

Systems frequently contaminated by stochastic noise in practice plus development in control and stability analysis of stochastic nonlinear systems [16], [17], [18] motivate us to consider the problem of controlling stochastic nonholonomic systems. In comparison with deterministic systems, stochastic nonholonomic systems have received much less attention. This is mainly due to appearance of Hessian terms in the infinitesimal generator of a Lyapunov function if the powerful Lyapunov direct method is used for control design. The Hessian terms cause difficulties in design of control inputs to ensure that the infinitesimal generator negative definite. Moreover, nonholonomic constraints, especially the $x_{0}$-subsystem, i.e., the first equation of (1), create an obstacle in control design. By assuming that the $x_{0}$-subsystem is deterministic, there are several works on design of asymptotic stabilizers in probability for stochastic nonholonomic systems, see [19] where unknown noise covariance is considered, and [20] where nonlinear appearance of unknown parameters is treated. These works are based on the input-to-state scaling proposed in [1], and the control design techniques for high order nonholonomic systems in power chained form in [21] and nonlinear systems with nonlinear appearance of unknown parameters in [2]. When the $x_{0}$-subsystem is also stochastic, there are some results available in [22] where the results are incorrect, and in [23] where the $x_{0}$-subsystem is linear.

The controllers in all of the above works on both deterministic and stochastic nonholonomic systems are not optimal in the sense that no meaningful cost function is resulted from their control designs. The aforementioned issues motivate contributions of this paper on design of optimal control inputs $u_{0}$ and $u_{1}$ with respect to a meaningful cost function to globally asymptotically stabilize the system (1) at the origin in probability. In particular, this paper addresses the following control objective:

Control Objective 1.1: Design the control inputs $u_{0}=\varpi_{0}\left(x_{0}\right)$ and $u_{1}=\varpi_{1}\left(x_{0}, \boldsymbol{x}\right)$ such that they guarantee global asymptotic stability in probability of the equilibrium $x_{0}=0$ and $\boldsymbol{x}=0$ and minimize a 
meaningful cost functional of the form

$$
J(\overline{\boldsymbol{u}})=\mathbb{E}\left\{\int_{t_{0}}^{\infty}\left(l(\overline{\boldsymbol{x}}(t))+\sigma\left(\left\|\boldsymbol{R}^{1 / 2}(\overline{\boldsymbol{x}}(t)) \overline{\boldsymbol{u}}(t)\right\|\right)\right) d t\right\},
$$

where $\overline{\boldsymbol{u}}=\operatorname{col}\left(u_{0}, u_{1}\right), \overline{\boldsymbol{x}}=\operatorname{col}\left(x_{0}, \boldsymbol{x}\right), l(\bullet)$ is a positive definite radially unbounded function, $\sigma(\bullet)$ is a class $\mathcal{K}_{\infty}$ function such that its derivative with respect to $\bullet$ is also a class $\mathcal{K}_{\infty}$ function, and $\boldsymbol{R}(\bullet)$ is a matrix-valued function satisfying $\boldsymbol{R}(\bullet)=\boldsymbol{R}^{T}(\bullet)>0$.

\section{PRELIMINARIES}

\section{A. Legendre-Fenchel transform}

Lemma 2.1: (Krstic and Li [24]) Let $\ell \sigma(\chi)$ denote the Legendre-Fenchel transform defined by

$$
\ell \sigma(\chi)=\chi\left(\sigma^{\star}\right)^{-1}(\chi)-\sigma\left(\left(\sigma^{\star}\right)^{-1}(\chi)\right)
$$

where $\sigma: \mathbb{R} \rightarrow \mathbb{R}$ is a class $\mathcal{K}_{\infty}$ function whose derivative $\sigma^{\star}(\chi)=\frac{d \sigma(\chi)}{d \chi}$ is also a class $\mathcal{K}_{\infty}$ function, and $\left(\sigma^{\star}\right)^{-1}(\chi)$ denotes the inverse function of $\sigma^{\star}(\chi)$. The Legendre-Fenchel transform $\ell \sigma(\chi)$ has the following properties

1) $\ell \sigma(\chi)=\int_{0}^{\chi}\left(\sigma^{\star}\right)^{-1}(s) d s$

2) $\ell \ell \sigma(\chi)=\sigma(\chi)$;

3) $\ell \sigma(\chi)$ is a class $\mathcal{K}_{\infty}$ function;

4) $\ell \sigma\left(\sigma^{\star}(\chi)\right)=\chi \sigma^{\star}(\chi)-\sigma(\chi)$.

\section{B. Young's inequality}

For $(x, y) \in \mathbb{R}^{2}$, the following Young inequality holds [25]:

$$
x y \leq \frac{\epsilon^{p}}{p}|x|^{p}+\frac{1}{q \epsilon^{q}}|y|^{q},
$$

where $\epsilon$ is a positive constant, and the constants $p>1$ and $q>1$ satisfy $(p-1)(q-1)=1$.

C. Solution of a linear time-varying stochastic system

Lemma 2.2: Consider the scalar linear time-varying stochastic system

$$
d x=(a(t) x+b(t)) d t+x \sum_{i=1}^{r} c_{i}(t) d w_{i},
$$

where $a(t), b(t)$ and $c_{i}(t)$ are real-valued Borel measurable bounded functions for $t \geq t_{0}$ and $w_{i}(t)$ is a standard Wiener process. Assume that the system (9) has a unique solution. Then this solution is given by

$$
x(t)=\phi(t)\left(x\left(t_{0}\right)+\int_{t_{0}}^{t} \frac{1}{\phi(s)} b(s) d s\right) .
$$

where

$$
\phi(t)=\exp \left[\int_{t_{0}}^{t}\left(\left(a(s)-\frac{1}{2} \sum_{i=1}^{r} c_{i}^{2}(s)\right) d s+\sum_{i=1}^{r} c_{i}(s) d w_{i}(s)\right)\right] .
$$

Proof. See Appendix A.

\section{Nonlinear stochastic systems}

Consider the nonlinear stochastic system

$$
d \boldsymbol{x}=\boldsymbol{f}(\boldsymbol{x}) d t+\boldsymbol{G}(\boldsymbol{x}) d \boldsymbol{w},
$$

where $\boldsymbol{x} \in \mathbb{R}^{n}$ is the state; and $\boldsymbol{f}: \mathbb{R}^{n} \rightarrow \mathbb{R}^{n}$ and $G: \mathbb{R}^{n} \rightarrow \mathbb{R}^{n \times r}$ are measurable on the given probability space $(\Omega, \mathcal{F}, \mathcal{P})$ with respect to the fixed $r$-dimensional independent standard Wiener process $\boldsymbol{w}$ and the independent initial condition $\boldsymbol{x}_{0}$ at $t_{0} \geq 0$ over this probability space. Moreover, $\boldsymbol{f}(\boldsymbol{x})$ and $\boldsymbol{G}(\boldsymbol{x})$ are locally Lipschitz and satisfy $\boldsymbol{f}(0)=0$ and $\boldsymbol{G}(0)=0$. 
1) Stochastic differentiation and infinite generator: Consider the nonlinear stochastic system (12) and let $y: \mathbb{R}^{n} \rightarrow \mathbb{R}$. Then the stochastic differentiation of $y(\boldsymbol{x}(t))$ is given by [26]:

$$
d y(\boldsymbol{x})=\frac{\partial y}{\partial \boldsymbol{x}} d \boldsymbol{x}+\frac{1}{2} \operatorname{Tr}\left(\boldsymbol{G}^{T}(\boldsymbol{x}) \frac{\partial^{2} y}{\partial \boldsymbol{x}^{2}} \boldsymbol{G}(\boldsymbol{x})\right) d t
$$

where $\operatorname{Tr}(\bullet)$ denotes the trace operator of $\bullet$.

For the nonlinear stochastic system (12), the infinite generator $\mathcal{L} V(\boldsymbol{x})$ of a $\mathcal{C}^{2}$ function $V(\boldsymbol{x})$ is defined as

$$
\mathcal{L} V(\boldsymbol{x})=\frac{\partial V}{\partial \boldsymbol{x}} \boldsymbol{f}(\boldsymbol{x})+\frac{1}{2} \operatorname{Tr}\left(\boldsymbol{G}^{T}(\boldsymbol{x}) \frac{\partial^{2} V}{\partial \boldsymbol{x}^{2}} \boldsymbol{G}(\boldsymbol{x})\right) .
$$

2) Stability in probability:

Definition 2.1: (Karatzas and Shreve [26]) The vector-valued function $\boldsymbol{x}(t)$ is called a strong solution of the nonlinear stochastic system (12) if it satisfies

1) $\boldsymbol{x}$ is adapted to the filtration $\left(\mathcal{G}_{t}\right)$, where $\mathcal{G}_{t}^{t_{0}}:=\max \left(\boldsymbol{G}(\boldsymbol{w}(s)), \boldsymbol{G}\left(\boldsymbol{x}\left(t_{0}\right)\right)\right.$ for all $t_{0} \leq s \leq t$ and $\mathcal{G}_{t}$ is the completion of $\bigcap_{s>t} \mathcal{G}_{s}^{t_{0}}$ with $\mathcal{P}$-null set [26];

2) $\boldsymbol{x}$ is a continuous process;

3) $\mathcal{P}\left(\boldsymbol{x}\left(t_{0}\right)=\boldsymbol{x}_{0}\right)=1$;

4) $\mathcal{P}\left(\int_{t_{0}}^{t}\left(\|\boldsymbol{f}(\boldsymbol{x}(s))\|+\|\boldsymbol{G}(\boldsymbol{x}(s))\|^{2}\right) d s<\infty\right)=1$ holds for all $t \geq t_{0} \geq 0$;

5) with probability one, we have

$$
\boldsymbol{x}(t)=\boldsymbol{x}\left(t_{0}\right)+\int_{t_{0}}^{t} \boldsymbol{f}(\boldsymbol{x}(s)) d s+\int_{t_{0}}^{t} \boldsymbol{G}(\boldsymbol{x}(s)) d \boldsymbol{w}(s),
$$

for all $t \geq t_{0} \geq 0$.

Definition 2.2: (Khasminskii [18], Krstic and Deng [17]) The equilibrium $\boldsymbol{x}=0$ of the system (12) is

- globally stable in probability if for all $\epsilon>0$ there exists a class $\mathcal{K}$ function $\sigma(\cdot)$ such that

$$
\mathcal{P}\left\{\|\boldsymbol{x}(t)\| \leq \sigma\left(\left\|\boldsymbol{x}\left(t_{0}\right)\right\|\right)\right\} \geq 1-\epsilon, \quad \forall t \geq t_{0} \geq 0, \forall \boldsymbol{x}\left(t_{0}\right) \in \mathbb{R}^{n} \backslash\{0\},
$$

- globally asymptotically stable in probability if for all $\epsilon>0$ there exists a class $\mathcal{K} \mathcal{L}$ function $\beta(\cdot, \cdot)$ such that

$$
\mathcal{P}\left\{\|\boldsymbol{x}(t)\| \leq \beta\left(\left\|\boldsymbol{x}\left(t_{0}\right)\right\|, t-t_{0}\right)\right\} \geq 1-\epsilon, \quad \forall t \geq t_{0} \geq 0, \forall \boldsymbol{x}\left(t_{0}\right) \in \mathbb{R}^{n} \backslash\{0\} .
$$

Theorem 2.1: (Krstic and Deng [17]) Suppose that there exist a $\mathcal{C}^{2}$ function $V(\boldsymbol{x})$, class $\mathcal{K}_{\infty}$ functions $\sigma_{1}$ and $\sigma_{2}$, and a class $\mathcal{K}$ function $\sigma_{3}$ such that

$$
\begin{aligned}
& \sigma_{1}(\|\boldsymbol{x}\|) \leq V(\boldsymbol{x}) \leq \sigma_{2}(\|\boldsymbol{x}\|), \\
& \mathcal{L} V(\boldsymbol{x})=\frac{\partial V(\boldsymbol{x})}{\partial \boldsymbol{x}} \boldsymbol{f}(\boldsymbol{x})+\frac{1}{2} \operatorname{Tr}\left\{\boldsymbol{G}^{T}(\boldsymbol{x}) \frac{\partial^{2} V(\boldsymbol{x})}{\partial \boldsymbol{x}^{2}} \boldsymbol{G}(\boldsymbol{x})\right\} \leq-\sigma_{3}(\|\boldsymbol{x}\|)+\varepsilon_{0},
\end{aligned}
$$

where $\varepsilon_{0}$ is a positive constant. Then there exists a unique strong solution of the system (12) for each $\boldsymbol{x}\left(t_{0}\right) \in \mathbb{R}^{n}$. Moreover, if $\varepsilon_{0}=0$ then the equilibrium $\boldsymbol{x}=0$ is globally asymptotically stable in probability, and the solution $\boldsymbol{x}(t)$ satisfies $\mathcal{P}\left\{\lim _{t \rightarrow \infty} \sigma_{3}(\|\boldsymbol{x}(t)\|)=0\right\}=1, \forall \boldsymbol{x}\left(t_{0}\right) \in \mathbb{R}^{n}$.

3) Inverse optimal stabilizer in probability: Consider the nonlinear stochastic system

$$
d \boldsymbol{x}=\boldsymbol{f}(\boldsymbol{x}) d t+\boldsymbol{G}_{1}(\boldsymbol{x}) d \boldsymbol{w}+\boldsymbol{G}_{2}(\boldsymbol{x}) \boldsymbol{u} d t,
$$

where $\boldsymbol{x} \in \mathbb{R}^{n}$ is the state, $\boldsymbol{u} \in \mathbb{R}^{m}$ is the control input, $\boldsymbol{w}$ is an $r$-dimensional independent standard Wiener process, $\boldsymbol{f}: \mathbb{R}^{n} \rightarrow \mathbb{R}^{n}$ and $\boldsymbol{G}_{1}: \mathbb{R}^{n} \rightarrow \mathbb{R}^{n \times r}$ are locally Lipschitz and satisfy $\boldsymbol{f}(0)=0$ and $\boldsymbol{G}_{1}(0)=0$, and $\boldsymbol{G}_{2}: \mathbb{R}^{n} \rightarrow \mathbb{R}^{n \times m}$.

Theorem 2.2: (Krstic and Deng [17]) Consider the control law

$$
\boldsymbol{u}^{\diamond}(\boldsymbol{x}(t))=-\boldsymbol{R}_{2}^{-1}\left(L_{\boldsymbol{G}_{2}} V\right)^{T} \frac{\ell \sigma\left(\left\|L_{\boldsymbol{G}_{2}} V \boldsymbol{R}_{2}^{-1 / 2}\right\|\right)}{\left\|L_{\boldsymbol{G}_{2}} V \boldsymbol{R}_{2}^{-1 / 2}\right\|^{2}},
$$


where $V(\boldsymbol{x})$ is a Lyapunov function candidate, $\sigma$ is a class $\mathcal{K}_{\infty}$ function whose derivative $\sigma^{\star}$ is also a class $\mathcal{K}_{\infty}$ function, $L_{\boldsymbol{G}_{2}} V=\frac{\partial V}{\partial \boldsymbol{x}} \boldsymbol{G}_{2}$ is a vector-valued function of $\boldsymbol{x}(t)$, and $\boldsymbol{R}_{2}(\boldsymbol{x})$ is a matrix-valued function of $\boldsymbol{x}(t)$ such that $\boldsymbol{R}_{2}(\boldsymbol{x})=\boldsymbol{R}_{2}^{T}(\boldsymbol{x})>0$. If the control law (20) globally asymptotically stabilizes the system (19) in probability at the origin with respect to $V(\boldsymbol{x})$, then the control law

$$
\boldsymbol{u}^{*}(\boldsymbol{x}(t))=-\frac{\beta}{2} \boldsymbol{R}_{2}^{-1}\left(L_{\boldsymbol{G}_{2}} V\right)^{T} \frac{\left(\sigma^{\star}\right)^{-1}\left(\left\|L_{\boldsymbol{G}_{2}} V \boldsymbol{R}_{2}^{-1 / 2}\right\|\right)}{\left\|L_{\boldsymbol{G}_{2}} V \boldsymbol{R}_{2}^{-1 / 2}\right\|}, \quad \beta \geq 2
$$

also globally asymptotically stabilizes the system (19) in probability at the origin, and minimizes the cost functional

$$
J(\boldsymbol{u})=\mathbb{E}\left\{\int_{0}^{\infty}\left[l(\boldsymbol{x})+\beta^{2} \sigma\left(\frac{2}{\beta}\left\|\boldsymbol{R}_{2}^{1 / 2} \boldsymbol{u}\right\|\right)\right] d t\right\}
$$

where

$$
l(\boldsymbol{x}(t))=2 \beta\left[\ell \sigma\left(\left\|L_{\boldsymbol{G}_{2}} V \boldsymbol{R}_{2}^{-1 / 2}\right\|\right)-L_{\boldsymbol{f}} V-\frac{1}{2} \operatorname{Tr}\left\{\boldsymbol{G}_{1}^{T} \frac{\partial^{2} V}{\partial \boldsymbol{x}^{2}} \boldsymbol{G}_{1}\right\}\right]+\beta(\beta-2) \ell \sigma\left(\left\|L_{\boldsymbol{G}_{2}} V \boldsymbol{R}_{2}^{-1 / 2}\right\|\right),
$$

with $L_{\boldsymbol{f}} V=\frac{\partial V}{\partial \boldsymbol{x}} \boldsymbol{f}$.

Remark 2.1: Since $\ell \sigma(\bullet)$ is also a class $\mathcal{K}_{\infty}$ function of $\bullet$ and $\boldsymbol{R}_{2}(\boldsymbol{x})=\boldsymbol{R}_{2}^{T}(\boldsymbol{x})>0$, the matrix

$$
\boldsymbol{M}_{2}(\boldsymbol{x})=\boldsymbol{R}_{2}^{-1} \frac{\ell \sigma\left(\left\|L_{\boldsymbol{G}_{2}} V \boldsymbol{R}_{2}^{-1 / 2}\right\|\right)}{\left\|L_{\boldsymbol{G}_{2}} V \boldsymbol{R}_{2}^{-1 / 2}\right\|^{2}}
$$

satisfies $\boldsymbol{M}_{2}(\boldsymbol{x})=\boldsymbol{M}_{2}^{T}(\boldsymbol{x})>0$. Therefore, Theorem 2.2 suggests a search for a control $\boldsymbol{u}^{\diamond}$, which globally asymptotically stabilizes the system (19) in probability, and has the form

$$
\boldsymbol{u}^{\diamond}(\boldsymbol{x}(t))=-\boldsymbol{M}(\boldsymbol{x})\left(L_{\boldsymbol{G}_{2}} V\right)^{T},
$$

where $\boldsymbol{M}(\boldsymbol{x})=\boldsymbol{M}^{T}(\boldsymbol{x})>0$. This control is referred to as anverse pre-optimal control law.

\section{CONTROL DESIGN: $x_{0}$-SUBSYSTEM}

To design the control $u_{0}$ that globally asymptotically stabilizes the $x_{0}$-subsystem in probability at the origin, we consider the following Lyapunov function candidate

$$
V_{0}\left(x_{0}\right)=\gamma_{0}\left(\frac{x_{0}^{2 k}}{2 k}\right)
$$

where $k \geq 2$ is an integer, and $\gamma_{0}\left(\frac{x_{0}^{2 k}}{2 k}\right)$ is a class $\mathcal{K}_{\infty}$ function of $\frac{x_{0}^{2 k}}{2 k}$. The class $\mathcal{K}_{\infty}$ function $\gamma_{0}\left(\frac{x_{0}^{2 k}}{2 k}\right)$ possesses the following properties for all $x_{0} \in \mathbb{R}$

$$
\begin{aligned}
& 0<\gamma_{0}^{\prime} \leq a_{0}, \\
& \Delta_{0}\left(x_{0}\right)=\gamma_{0}^{\prime \prime} x_{0}^{2 k}+\gamma_{0}^{\prime}(2 k-1)>0,
\end{aligned}
$$

where $\gamma_{0}^{\prime}=\partial \gamma_{0} / \partial\left(\frac{x_{0}^{2 k}}{2 k}\right), \gamma_{0}^{\prime \prime}=\partial^{2} \gamma_{0} / \partial\left(\frac{x_{0}^{2 k}}{2 k}\right)^{2}$, and $a_{0}$ is a positive constant. It should not be confusing between the notation ' here and ${ }^{\star}$ used in Theorem 2.2, as such $\gamma_{0}^{\star}=d \gamma_{0} / d x_{0}=\gamma_{0}^{\prime} \partial\left(\frac{x_{0}^{2 k}}{2 k}\right) / \partial x_{0}=\gamma_{0}^{\prime} x_{0}^{2 k-1}$.

Remark 3.1:

1) Different choice of the class $\mathcal{K}_{\infty}$ function $\gamma_{0}$ in (26) allows different strength of nonlinearity of the Lyapunov function candidate $V_{0}$, i.e., $V_{0}$ can be either nonlinearly weak or nonlinearly strong. Nonlinearly weak Lyapunov function candidates have a potential application in control of underactuated stochastic mechanical systems as these systems usually require Lyapunov functions of a nonlinearly weak form even in deterministic cases [27], [28], [29]. Examples of $\gamma_{0}(\bullet)$ are $\gamma_{0}(\bullet)=\bullet$ and $\gamma_{0}(\bullet)=\sqrt{1+\bullet}-1$. Indeed, a quartic form proposed in [30], [31] is of a special case of (26).

2) The condition $k \geq 2$ eases the calculation of the upper-bound of the Hessian term of the infinitesimal generator $\mathcal{L} V_{0}\left(x_{0}\right)$ in the control design later. 
From (26) and the first equation of (1), applying the formula (14) results in $\mathcal{L} V_{0}\left(x_{0}\right)$ as

$$
\begin{aligned}
\mathcal{L} V_{0}\left(x_{0}\right) & =\frac{\partial V_{0}}{\partial x_{0}} u_{0}+\frac{1}{2} \operatorname{Tr}\left(\boldsymbol{\varphi}_{0}\left(x_{0}\right) \frac{\partial^{2} V_{0}}{\partial x_{0}^{2}} \boldsymbol{\varphi}_{0}^{T}\left(x_{0}\right)\right) \\
& =\gamma_{0}^{\prime} x_{0}^{2 k-1} u_{0}+\frac{1}{2}\left(\gamma_{0}^{\prime}(2 k-1) x_{0}^{2 k-2}+\gamma_{0}^{\prime \prime} x_{0}^{2(2 k-1)}\right)\left\|\boldsymbol{\varphi}_{0}\left(x_{0}\right)\right\|^{2} \\
& =\gamma_{0}^{\prime} x_{0}^{2 k-1} u_{0}+\frac{1}{2} \Delta_{0}\left(x_{0}\right) x_{0}^{2(k-1)}\left\|\boldsymbol{\varphi}_{0}\left(x_{0}\right)\right\|^{2},
\end{aligned}
$$

where $\Delta_{0}\left(x_{0}\right)$ is defined in (27). Assumption 1.1 on $\varphi_{0}\left(x_{0}\right)$ implies from the mean-value theorem that there exists a smooth vector function $\varphi_{00}\left(x_{0}\right)$ such that

$$
\varphi_{0}\left(x_{0}\right)=x_{0} \varphi_{00}\left(x_{0}\right) .
$$

Substituting (29) into the last equation of (28) results in

$$
\mathcal{L} V_{0}\left(x_{0}\right)=\gamma_{0}^{\prime} x_{0}^{2 k-1}\left(u_{0}+x_{0} p_{0}\left(x_{0}\right)\right),
$$

where

$$
p_{0}\left(x_{0}\right)=\frac{1}{2 \gamma_{0}^{\prime}} \Delta_{0}\left(x_{0}\right)\left\|\varphi_{00}\left(x_{0}\right)\right\|^{2},
$$

which has no problem with $\gamma_{0}^{\prime}$ in the denominator since Properties of $\gamma_{0}$ in (27) ensure that $\gamma_{0}^{\prime}>0$ for all $x_{0} \in \mathbb{R}$. Since $x_{0}=0$ is the equilibrium point of the $x_{0}$-subsystem, the control $u_{0}(t)$ is expected to be zero for all $t \geq t_{0} \geq 0$ if $x_{0}\left(t_{0}\right)=0$. However, $u_{0}(t)=0$ for all $t \geq t_{0} \geq 0$ results in uncontrollability of the $\boldsymbol{x}$-subsystem. Our goal is to achieve $\lim _{t \rightarrow \infty} u_{0}(t)=0$ and globally asymptotically stabilizes the $x_{0}$-subsystem simultaneously. Therefore, we consider two cases $x_{0}\left(t_{0}\right) \neq 0$ and $x_{0}\left(t_{0}\right)=0$.

\section{A. Design of inverse pre-optimal control $u_{0}^{\diamond}$}

1) Case $x_{0}\left(t_{0}\right) \neq 0$ : Motivated by Remark 2.1, the control $u_{0}^{\diamond}$, which is a modified type of Sontag's formula [32], is designed from (30) as follows

$$
u_{0}^{\diamond}=-\left(k_{0}+\sqrt{c_{0}+p_{0}^{2}\left(x_{0}\right)}\right) x_{0}:=\vartheta_{0}^{\diamond}\left(x_{0}\right),
$$

where $c_{0}$ and $k_{0}$ are positive constants. The constant $k_{0}$ is chosen such that

$$
k_{0}>\frac{2 k-1}{2 k} \epsilon^{\frac{2 k}{2 k-1}} a_{0}^{\frac{1}{2 k-1}}+2 \epsilon,
$$

with $\epsilon$ being a strictly positive constant. This choice of $k_{0}$ is to be used in stability analysis of the $\boldsymbol{x}$ subsystem in the next sections. Substituting (32) into (30) results in

$$
\left.\mathcal{L} V_{0}\left(x_{0}\right)\right|_{(32)}=-\gamma_{0}^{\prime} x_{0}^{2 k}\left(k_{0}+\sqrt{c_{0}+p_{0}^{2}\left(x_{0}\right)}-p_{0}\left(x_{0}\right)\right) \leq-k_{0} \gamma_{0}^{\prime} x_{0}^{2 k} .
$$

It will be shown in Theorem 3.1 and Theorem 5.1 that the inverse pre-optimal control $u_{0}^{\diamond}$ given by (32) achieves global asymptotic stabilization of the $x_{0}$-subsystem and can be amended to achieve optimality.

2) Case $x_{0}\left(t_{0}\right)=0$ : The control $u_{0}^{\diamond}$ is designed such that it first drives the state $x_{0}(t)$ of the $x_{0}$-subsystem away from zero but still keeps this subsystem well-defined then forces the state $x_{0}(t)$ asymptotically converge to zero. As such, the procedure to design the control $u_{0}^{\diamond}$ as follows:

Procedure 3.1:

1) If $\left|\vartheta_{0}^{\diamond}\left(x_{0}\right)\right| \leq \delta_{0}$ with $\vartheta_{0}\left(x_{0}\right)$ being defined in (32) and $\delta_{0}$ being a small positive constant (theoretically $\delta_{0}$ can be chosen to be zero), then the control $u_{0}^{\diamond}$ is chosen as

$$
u_{0}^{\diamond}=-\left(k_{0}+\sqrt{c_{0}+p_{0}^{2}\left(x_{0}\right)}\right) x_{0}+\eta_{0},
$$

where $\eta_{0}$ is a positive constant and satisfies $\eta_{0}>\delta_{0}$. 
2) If $\left|\vartheta_{0}^{\diamond}\left(x_{0}\right)\right|>\delta_{0}$, the control $u_{0}^{\diamond}$ is switched from (35) to

$$
u_{0}^{\diamond}=-\left(k_{0}+\sqrt{c_{0}+p_{0}^{2}\left(x_{0}\right)}\right) x_{0} .
$$

The above control design procedure is interpreted as follows. At the initial time $t_{0}, x_{0}\left(t_{0}\right)=0$, the control $u_{0}^{\diamond}$ given by (35) drives the state $x_{0}(t)$ away from zero. Since $\eta_{0}>\delta_{0}$, we have $u_{0}^{\diamond} \geq \eta_{0}-\delta_{0}>0$. Having driven the state $x_{0}(t)$ away from zero, the control $u_{0}^{\diamond}$ is switched to (36) to forces the state $x_{0}(t)$ to asymptotically converge to zero in probability. Let $t_{s}^{\diamond}$ denote the time when $u_{0}^{\diamond}$ is switched from (35) to (36). With the above control design procedure, the infinitesimal generator (30) can be written as

$$
\begin{cases}\left.\mathcal{L} V_{0}\left(x_{0}\right)\right|_{(35)}=-\gamma_{0}^{\prime} x_{0}^{2 k}\left(k_{0}+\sqrt{c_{0}+p_{0}^{2}\left(x_{0}\right)}-p_{0}\left(x_{0}\right)\right)+\gamma_{0}^{\prime} x_{0}^{2 k-1} \eta_{0}, & \text { for } t \leq t_{s}^{\diamond}, \\ \left.\mathcal{L} V_{0}\left(x_{0}\right)\right|_{(36)}=-\gamma_{0}^{\prime} x_{0}^{2 k}\left(k_{0}+\sqrt{c_{0}+p_{0}^{2}\left(x_{0}\right)}-p_{0}\left(x_{0}\right)\right), & \text { elsewhere. }\end{cases}
$$

Applying Young's inequality (8) to the term $\gamma_{0}^{\prime} x_{0}^{2 k-1} \eta_{0}$ yields

$$
\gamma_{0}^{\prime} x_{0}^{2 k-1} \eta_{0} \leq \varepsilon_{1}\left(\gamma_{0}^{\prime}\right)^{\frac{1}{2 k-1}} \gamma_{0}^{\prime} x_{0}^{2 k}+\varepsilon_{2} \eta_{0}^{2 k}
$$

where

$$
\varepsilon_{1}=\frac{2 k-1}{2 k} \epsilon^{\frac{2 k}{2 k-1}}, \quad \varepsilon_{2}=\frac{1}{2 k \epsilon^{2 k}} .
$$

Substituting (38) into (37) with a note that $\gamma_{0}^{\prime} \leq a_{0}$, see (27), yields

$$
\begin{cases}\left.\mathcal{L} V_{0}\left(x_{0}\right)\right|_{(35)} \leq-\left(k_{0}-\varepsilon_{1} a_{0}^{\frac{1}{2 k-1}}\right) \gamma_{0}^{\prime} x_{0}^{2 k}+\varepsilon_{2} \eta_{0}^{2 k}, & \text { for } t \leq t_{s}^{\diamond}, \\ \left.\mathcal{L} V_{0}\left(x_{0}\right)\right|_{(36)} \leq-k_{0} \gamma_{0}^{\prime} x_{0}^{2 k}, & \text { elsewhere }\end{cases}
$$

\section{B. Design of inverse optimal control $u_{0}^{*}$}

1) Case $x_{0}\left(t_{0}\right) \neq 0$ : Applying Theorem 2.2 to the first equation of (1) results in the inverse optimal control $u_{0}^{*}$ as follows

$$
u_{0}^{*}=-\frac{\beta_{0}}{2} R_{0}^{-1} \gamma_{0}^{\prime} x_{0}^{2 k-1} \frac{\left(\sigma_{0}^{\star}\right)^{-1}\left(\left|R_{0}^{-1 / 2} \gamma_{0}^{\prime} x_{0}^{2 k-1}\right|\right)}{\left|R_{0}^{-1 / 2} \gamma_{0}^{\prime} x_{0}^{2 k-1}\right|},
$$

where $\beta_{0} \geq 2, R_{0}\left(x_{0}\right)$ is a positive definite function of $x_{0}, \sigma_{0}$ is a class $\mathcal{K}_{\infty}$ function, $\sigma_{0}^{\star}(\bullet)=\frac{d \sigma_{0}}{d \bullet}$, and the notation $\left(\sigma_{0}^{\star}\right)^{-1}(\bullet)$ denotes the inverse function of $\sigma_{0}^{\star}$. The class $\mathcal{K}_{\infty}$ function $\sigma_{0}$ is chosen by the designer.

We now determine $R_{0}\left(x_{0}\right)$. By Theorem 2.2, the control $u_{0}^{\diamond}$, which stabilizes the first equation of (1), should be of the form (20), i.e.,

$$
u_{0}^{\diamond}=-R_{0}^{-1} \gamma_{0}^{\prime} x_{0}^{2 k-1} \frac{\ell \sigma_{0}\left(\left|R_{0}^{-1 / 2} \gamma_{0}^{\prime} x_{0}^{2 k-1}\right|\right)}{\left|R_{0}^{-1 / 2} \gamma_{0}^{\prime} x_{0}^{2 k-1}\right|^{2}} .
$$

The positive definite function $R_{0}\left(x_{0}\right)$ is to be determined so that the control $u_{0}^{\diamond}$ given in (32) is the same with the one given in (42). As such, comparing (42) with (32) results in the fact that $R_{0}\left(x_{0}\right)$ needs to satisfy the following equation

$$
R_{0}^{-1} \gamma_{0}^{\prime} x_{0}^{2 k-1} \frac{\ell \sigma_{0}\left(\left|R_{0}^{-1 / 2} \gamma_{0}^{\prime} x_{0}^{2 k-1}\right|\right)}{\left|R_{0}^{-1 / 2} \gamma_{0}^{\prime} x_{0}^{2 k-1}\right|^{2}}=\left(k_{0}+\sqrt{c_{0}+p_{0}^{2}\left(x_{0}\right)}\right) x_{0}
$$

for all $x_{0} \in \mathbb{R}$. The equation (43) is equivalent to $\ell \sigma_{0}\left(\left|R_{0}^{-1 / 2} \gamma_{0}^{\prime} x_{0}^{2 k-1}\right|\right)=\gamma_{0}^{\prime} x_{0}^{2 k}\left(k_{0}+\sqrt{c_{0}+p_{0}^{2}\left(x_{0}\right)}\right)$, which gives

$$
R_{0}\left(x_{0}\right)=\left[\frac{\left|\gamma_{0}^{\prime} x_{0}^{2 k-1}\right|}{\left(\ell \sigma_{0}\right)^{-1}\left(\gamma_{0}^{\prime} x_{0}^{2 k}\left(k_{0}+\sqrt{c_{0}+p_{0}^{2}\left(x_{0}\right)}\right)\right)}\right]^{2},
$$


where the notation $\left(\ell \sigma_{0}\right)^{-1}(\bullet)$ denotes the inverse function of $\ell \sigma_{0}(\bullet)$. The function $R_{0}\left(x_{0}\right)$ given by (44) is continuous away from the origin and positive definite for all $x_{0} \in \mathbb{R}$. Substituting $R_{0}\left(x_{0}\right)$ given in (44) into (41) yields an explicit expression of the optimal control $u_{0}^{*}$, see Appendix B, as

$$
u_{0}^{*}=-\frac{\beta_{0}}{2}\left[\left(k_{0}+\sqrt{c_{0}+p_{0}^{2}\left(x_{0}\right)}\right)+R_{0}^{-1} \gamma_{0}^{\prime} x_{0}^{2 k-2} \frac{\sigma_{0}\left(\left(\sigma_{0}^{\star}\right)^{-1}\left(\left|R_{0}^{-1 / 2} \gamma_{0}^{\prime} x_{0}^{2 k-1}\right|\right)\right)}{\left|R_{0}^{-1 / 2} \gamma_{0}^{\prime} x_{0}^{2 k-1}\right|^{2}}\right] x_{0}:=\vartheta_{0}^{*}\left(x_{0}\right) .
$$

Substituting (45) into (30) yields

$$
\begin{aligned}
\left.\mathcal{L} V_{0}\left(x_{0}\right)\right|_{(45)} & =-\gamma_{0}^{\prime} x_{0}^{2 k}\left[\frac{\beta_{0}}{2}\left(k_{0}+\sqrt{c_{0}+p_{0}^{2}\left(x_{0}\right)}\right)-p_{0}\left(x_{0}\right)+\frac{\beta_{0}}{2} R_{0}^{-1} \gamma_{0}^{\prime} x_{0}^{2 k-2} \frac{\sigma_{0}\left(\left(\sigma_{0}^{\star}\right)^{-1}\left(\left|R_{0}^{-1 / 2} \gamma_{0}^{\prime} x_{0}^{2 k-1}\right|\right)\right)}{\left|R_{0}^{-1 / 2} \gamma_{0}^{\prime} x_{0}^{2 k-1}\right|^{2}}\right] \\
& \leq-\frac{\beta_{0} k_{0}}{2} \gamma_{0}^{\prime} x_{0}^{2 k},
\end{aligned}
$$

where we have used the fact that $\beta_{0} \geq 2$ and $k \geq 2$.

2) Case $x_{0}\left(t_{0}\right)=0$ : Similarly to the design of the inverse pre-optimal control $u_{0}^{\diamond}$ in Procedure 3.1 , the inverse optimal control $u_{0}^{*}$ is chosen as in the following procedure.

Procedure 3.2:

- If $\left|\vartheta_{0}^{*}\left(x_{0}\right)\right| \leq \delta_{0}$ with $\vartheta_{0}^{*}\left(x_{0}\right)$ being defined in (45), the inverse optimal control $u_{0}^{*}$ is chosen as

$$
u_{0}^{*}=-\frac{\beta_{0}}{2}\left[\left(k_{0}+\sqrt{c_{0}+p_{0}^{2}\left(x_{0}\right)}\right)+R_{0}^{-1} \gamma_{0}^{\prime} x_{0}^{2 k-2} \frac{\sigma_{0}\left(\left(\sigma_{0}^{\star}\right)^{-1}\left(\left|R_{0}^{-1 / 2} \gamma_{0}^{\prime} x_{0}^{2 k-1}\right|\right)\right)}{\left|R_{0}^{-1 / 2} \gamma_{0}^{\prime} x_{0}^{2 k-1}\right|^{2}}\right] x_{0}+\eta_{0} .
$$

- If $\left|\vartheta_{0}^{*}\left(x_{0}\right)\right|>\delta_{0}$, the inverse optimal control $u_{0}^{*}$ is switched from (47) to

$$
u_{0}^{*}=-\frac{\beta_{0}}{2}\left[\left(k_{0}+\sqrt{c_{0}+p_{0}^{2}\left(x_{0}\right)}\right)+R_{0}^{-1} \gamma_{0}^{\prime} x_{0}^{2 k-2} \frac{\sigma_{0}\left(\left(\sigma_{0}^{\star}\right)^{-1}\left(\left|R_{0}^{-1 / 2} \gamma_{0}^{\prime} x_{0}^{2 k-1}\right|\right)\right)}{\left|R_{0}^{-1 / 2} \gamma_{0}^{\prime} x_{0}^{2 k-1}\right|^{2}}\right] x_{0} .
$$

Let $t_{s}^{*}$ denote the time when $u_{0}^{*}$ is switched from (47) to (48). With the above control design procedure and the use of the same techniques in derivation of (40) and (46), we can write (30) as

$$
\begin{cases}\left.\mathcal{L} V_{0}\left(x_{0}\right)\right|_{(47)} \leq-\left(k_{0}-\varepsilon_{1} a_{0}^{\frac{1}{2 k-1}}\right) \gamma_{0}^{\prime} x_{0}^{2 k}+\varepsilon_{2} \eta_{0}^{2 k}, & \text { for } t \leq t_{s}^{*}, \\ \left.\mathcal{L} V_{0}\left(x_{0}\right)\right|_{(48)} \leq-k_{0} \gamma_{0}^{\prime} x_{0}^{2 k}, & \text { elsewhere. }\end{cases}
$$

We now present the first result on stability and convergence of the $x_{0}$-subsystem. This result is crucial for the input-to-state scaling and the design of the control $u_{1}$ in the next sections.

Theorem 3.1: Under Assumption 1.1, for any initial value $x_{0}\left(t_{0}\right) \in \mathbb{R}$, the following results hold:

1) With the inverse pre-optimal control $u_{0}^{\diamond}$ given by (32) for the case $x_{0}\left(t_{0}\right) \neq 0$ or in Procedure 3.1 for the case $x_{0}\left(t_{0}\right)=0$, the solution $x_{0}(t)$ of the $x_{0}$-subsystem exists uniquely and asymptotically converges to zero in probability. Moreover, the control $u_{0}^{\diamond}$ does not cross zero and asymptotically converges to zero in probability.

2) Under an additional condition on $\sigma_{0}(\bullet)$ that $\sigma_{0}^{\star}(\bullet)$ is a class $\mathcal{K}_{\infty}$ function of $\bullet$, the inverse optimal control $u_{0}^{*}$ given by (41) for the case $x_{0}\left(t_{0}\right) \neq 0$ or in Procedure 3.2 for the case $x_{0}\left(t_{0}\right)=0$ guarantees existence, uniqueness and global asymptotic convergence to zero in probability of the solution $x_{0}(t)$ of the $x_{0}$-subsystem. In addition, the control $u_{0}^{*}$ does not cross zero and asymptotically converges to zero in probability. Moreover, the following cost functional is minimized:

$$
J_{0}\left(u_{0}\right)=\mathbb{E}\left\{\int_{t_{0}^{*}}^{\infty}\left[l_{0}\left(x_{0}\right)+\beta_{0}^{2} \sigma_{0}\left(\frac{2}{\beta_{0}}\left|R_{0}^{1 / 2} u_{0}\right|\right)\right] d t\right\}
$$


where $t_{0}^{*}=\max \left(t_{0}, t_{s}^{*}\right)$ and

$$
\left.l_{0}\left(x_{0}\right)=2 \beta_{0}\left[\ell \sigma_{0}\left(\left|\gamma_{0}^{\prime} x_{0}^{2 k-1} R_{0}^{-1 / 2}\right|\right)-\frac{1}{2} \Delta_{0}\left(x_{0}\right)\left\|\varphi_{0}\left(x_{0}\right)\right\|^{2}\right)\right]+\beta_{0}\left(\beta_{0}-2\right) \ell \sigma_{0}\left(\left|\gamma_{0}^{\prime} x_{0}^{2 k-1} R_{0}^{-1 / 2}\right|\right),
$$

with $\Delta_{0}\left(x_{0}\right)$ being given by (27).

Proof. See Appendix C.

\section{Example 1.1 (cont'd)}

We now continue Example 1.1. For illustration, we take $s=1$ and $\zeta_{0}\left(x_{0}, x_{1}, x_{2}\right)=x_{0}^{2}+x_{1}^{2}+x_{2}^{2}$. It is seen that the cart kinematic system (5) is of the form of (1) with $\varphi_{0}\left(x_{0}\right)=0, \varphi_{1}\left(x_{0}, x_{1}\right)=0$, and $\varphi_{2}\left(x_{0}, x_{1}, x_{2}\right)=x_{0}^{2}+x_{1}^{2}+x_{2}^{2}$. Here, we apply the control design in this section to design the control $u_{0}$, i.e, the first equation of (5) is considered. The illustration on the design of the control $u_{1}$ will be presented in subsections IV-B and V-C. As such, let $\sigma_{0}(\chi)=\frac{\chi^{4}}{4}$ and $\gamma_{0}(\chi)=\chi$. We then have

$$
\begin{aligned}
& \sigma_{0}^{\star}(\chi)=\chi^{3},\left(\sigma_{0}^{\star}\right)^{-1}(\chi)=\chi^{1 / 3}, \ell \sigma_{0}(\chi)=\frac{3}{4} \chi^{4 / 3},\left(\ell \sigma_{0}\right)^{-1}(\chi)=\left(\frac{4}{3} \chi\right)^{3 / 4}, \\
& \gamma_{0}^{\prime}=1, \gamma_{0}^{\prime \prime}=0, \Delta_{0}=2 k-1, \varphi_{00}\left(x_{0}\right)=0, p_{0}\left(x_{0}\right)=0 .
\end{aligned}
$$

With the above expressions, the function $R_{0}\left(x_{0}\right)$ is calculated from (44) as follows

$$
R_{0}\left(x_{0}\right)=\left[\frac{\left|x_{0}^{2 k-1}\right|}{\left(\frac{4}{3} x_{0}^{2 k}\left(k_{0}+\sqrt{c_{0}+p_{0}^{2}\left(x_{0}\right)}\right)\right)^{3 / 4}}\right]^{2}=\frac{\left|x_{0}^{k-2}\right|}{\left(\frac{4}{3}\left(k_{0}+\sqrt{c_{0}}\right)\right)^{3 / 2}} .
$$

For the case $x_{0}\left(t_{0}\right) \neq 0$, the inverse optimal control $u_{0}^{*}$ is calculated from (45) with $R_{0}\left(x_{0}\right)$ in (53) as follows:

$$
u_{0}^{*}=-\frac{2 \beta_{0}}{3}\left(k_{0}+\sqrt{c_{0}}\right) x_{0}:=\vartheta_{0}^{*}\left(x_{0}\right) .
$$

For the case $x_{0}\left(t_{0}\right)=0$, the inverse optimal control $u_{0}^{*}$ is calculated from Procedure 3.2 as follows:

If $\left|\vartheta_{0}^{*}\left(x_{0}\right)\right| \leq \delta_{0}$ with $\vartheta_{0}^{*}\left(x_{0}\right)$ being defined in (54), we have

$$
u_{0}^{*}=-\frac{2 \beta_{0}}{3}\left(k_{0}+\sqrt{c_{0}}\right) x_{0}+\eta_{0} .
$$

If $\left|\vartheta_{0}^{*}\left(x_{0}\right)\right|>\delta_{0}$, the inverse optimal control $u_{0}^{*}$ is switched from (55) to

$$
u_{0}^{*}=-\frac{2 \beta_{0}}{3}\left(k_{0}+\sqrt{c_{0}}\right) x_{0} .
$$

\section{INPUT-TO-STATE SCALING}

Having designed the inverse pre-optimal control $u_{0}^{\diamond}$ or the inverse optimal control $u_{0}^{*}$ possessing properties in Theorem 3.1, the remaining obstacle is the appearance of $u_{0}$ (or $u_{0}^{\diamond}$ or $u_{0}^{*}$ ) as a factor of each $x_{i}$, $i=1, \ldots, n-1$, see (1). Since $u_{0}$ (or $u_{0}^{\diamond}$ or $u_{0}^{*}$ ) asymptotically converges to zero in probability, the control $u_{1}$ needs to be designed such that $x_{i}, i=1, \ldots, n-1$ converges to zero faster than $u_{0}$ (or $u_{0}^{\diamond}$ or $u_{0}^{*}$ ) to avoid loss of controllability for the $\boldsymbol{x}$-subsystem. This is done by 1) introducing the input-to-state scaling [1] and 2) designing the control $u_{1}$ to globally asymptotically stabilize the scaled system in probability. In the rest of the paper, we consider the inverse optimal control $u_{0}^{*}$ since it is more efficient than the inverse pre-optimal control $u_{0}^{\diamond}$. 


\section{A. Input-to-state scaling for system (1)}

We introduce the following input-to-state scaling transformation

$$
z_{i}=\frac{x_{i}}{\left(u_{0}^{*}\right)^{n-i}}, \quad i=1, \ldots, n .
$$

Applying the stochastic differentiation formula (13) and using (1) result in

$$
\begin{aligned}
& d x_{0}=u_{0}^{*}\left(x_{0}\right) d t+\boldsymbol{\phi}_{0}^{T}\left(x_{0}\right) d \boldsymbol{w}, \\
& d z_{i}=\left(z_{i+1}+f_{i}\left(x_{0}, z_{i}\right)\right) d t+\boldsymbol{\phi}_{i}^{T}\left(x_{0}, \overline{\boldsymbol{z}}_{i}\right) d \boldsymbol{w}, \quad i=1, \ldots, n-1, \\
& d z_{n}=u_{1} d t+\boldsymbol{\phi}_{n}^{T}\left(x_{0}, \boldsymbol{z}\right) d \boldsymbol{w},
\end{aligned}
$$

where we have also rewritten $d x_{0}$ for convenience, the optimal control $u_{0}^{*}\left(x_{0}\right)$ is given in (45) for the case $x_{0}\left(t_{0}\right) \neq 0$ and in Procedure 3.2 for the case $x_{0}\left(t_{0}\right)=0, \bar{z}_{i}=\operatorname{col}\left(z_{1}, \ldots, z_{i}\right), \boldsymbol{z}=\operatorname{col}\left(z_{1}, \ldots, z_{n}\right)$, and the functions $\phi_{0}\left(x_{0}\right), f_{i}\left(x_{0}, z_{i}\right), \boldsymbol{\phi}_{i}\left(x_{0}, \overline{\boldsymbol{z}}_{i}\right)$, and $\boldsymbol{\phi}_{n}\left(x_{0}, \boldsymbol{z}\right)$ are given by

$$
\begin{aligned}
& f_{i}\left(x_{0}, z_{i}\right)=\frac{\partial z_{i}}{\partial u_{0}^{*}}\left(\frac{\partial u_{0}^{*}}{\partial x_{0}} u_{0}^{*}\left(x_{0}\right)+\frac{1}{2} \frac{\partial^{2} u_{0}^{*}}{\partial x_{0}^{2}}\left\|\boldsymbol{\varphi}_{0}\left(x_{0}\right)\right\|^{2}\right)+\frac{1}{2} \frac{\partial^{2} z_{i}}{\partial\left(u_{0}^{*}\right)^{2}}\left\|\frac{\partial u_{0}^{*}}{\partial x_{0}} \boldsymbol{\varphi}_{0}\left(x_{0}\right)\right\|^{2}, \\
& \boldsymbol{\phi}_{i}\left(x_{0}, \overline{\boldsymbol{z}}_{i}\right)=\frac{\partial z_{i}}{\partial x_{i}} \boldsymbol{\varphi}_{i}\left(x_{0}, u_{0}^{*}, \overline{\boldsymbol{x}}_{i}\right)+\frac{\partial z_{i}}{\partial u_{0}^{*}} \frac{\partial u_{0}^{*}}{\partial x_{0}} \boldsymbol{\varphi}_{0}\left(x_{0}\right), i=1, \ldots, n-1, \\
& \boldsymbol{\phi}_{0}\left(x_{0}\right)=\boldsymbol{\varphi}_{0}\left(x_{0}\right), \boldsymbol{\phi}_{n}\left(x_{0}, \boldsymbol{z}\right)=\boldsymbol{\varphi}_{n}\left(x_{0}, u_{0}^{*}, \boldsymbol{x}\right) .
\end{aligned}
$$

\section{B. Example 1.1 (cont'd)}

We now continue Example 1.1 to illustrate the input-to-state scaling developed in this section. Applying the input-to-state scaling (57) results in

$$
\begin{aligned}
& z_{1}=\frac{x_{1}}{u_{0}^{*}}, z_{2}=x_{2} \\
& \phi_{0}\left(x_{0}\right)=0, \phi_{1}\left(x_{0}, z_{1}\right)=0, \phi_{2}\left(x_{0}, z_{1}, z_{2}\right)=x_{0}^{2}+u_{0}^{* 2} z_{1}^{2}+z_{2}^{2}, f_{1}\left(x_{0}, z_{1}\right)=\frac{\partial z_{1}}{\partial u_{0}^{*}} \frac{\partial u_{0}^{*}}{\partial x_{0}} u_{0}^{*}\left(x_{0}\right) .
\end{aligned}
$$

\section{Control Design: $\boldsymbol{x}$-SUBSyStem}

A. Case $x_{0}\left(t_{0}\right) \neq 0$

1) Design of the stabilizing control $u_{1}$ and inverse pre-optimal control $u_{1}^{\diamond}$ : Define

$$
e_{i}=z_{i}-\alpha_{i-1}\left(x_{0}, \overline{\boldsymbol{z}}_{i-1}\right), \quad i=1, \ldots, n,
$$

where $\alpha_{i-1}\left(x_{0}, \bar{z}_{i-1}\right)$ is referred to as the virtual control of $z_{i}$, and $\alpha_{0}=0$. Applying the stochastic differentiation formula (13) to (61) and using (58) give

$$
\begin{aligned}
& d e_{i}=\left(e_{i+1}+\alpha_{i}\left(x_{0}, \overline{\boldsymbol{z}}_{i}\right)+\Omega_{i}\left(x_{0}, \overline{\boldsymbol{z}}_{i}\right)\right) d t+\boldsymbol{\lambda}_{i}^{T}\left(x_{0}, \overline{\boldsymbol{z}}_{i}\right) d \boldsymbol{w}, \quad i=1, \ldots, n-1, \\
& d e_{n}=\left(u_{1}+\Omega_{n}\left(x_{0}, \boldsymbol{z}\right)\right) d t+\boldsymbol{\lambda}_{n}^{T}\left(x_{0}, \boldsymbol{z}\right) d \boldsymbol{w}
\end{aligned}
$$

where

$$
\begin{aligned}
\Omega_{i}\left(x_{0}, \overline{\boldsymbol{z}}_{i}\right)= & f_{i}\left(x_{0}, z_{i}\right)-\frac{\partial \alpha_{i-1}\left(x_{0}, \overline{\boldsymbol{z}}_{i-1}\right)}{\partial x_{0}} u_{0}^{*}\left(x_{0}\right)-\frac{1}{2} \frac{\partial^{2} \alpha_{i-1}\left(x_{0}, \overline{\boldsymbol{z}}_{i-1}\right)}{\partial x_{0}^{2}}\left\|\boldsymbol{\phi}_{0}\left(x_{0}\right)\right\|^{2} \\
& -\sum_{p=1}^{i-1} \frac{\partial^{2} \alpha_{i-1}\left(x_{0}, \overline{\boldsymbol{z}}_{i-1}\right)}{\partial x_{0} \partial z_{p}} \boldsymbol{\phi}_{p}^{T}\left(x_{0}, \overline{\boldsymbol{z}}_{p}\right) \boldsymbol{\phi}_{0}\left(x_{0}\right)-\sum_{j=1}^{i-1} \frac{\partial \alpha_{i-1}\left(x_{0}, \overline{\boldsymbol{z}}_{i-1}\right)}{\partial z_{j}}\left(z_{j+1}+f_{j}\left(x_{0}, \overline{\boldsymbol{z}}_{j}\right)\right) \\
& -\frac{1}{2} \sum_{p=1, q=1}^{i-1} \frac{\partial^{2} \alpha_{i-1}\left(x_{0}, \overline{\boldsymbol{z}}_{i-1}\right)}{\partial z_{p} \partial z_{q}} \boldsymbol{\phi}_{p}^{T}\left(x_{0}, \overline{\boldsymbol{z}}_{p}\right) \boldsymbol{\phi}_{q}\left(x_{0}, \overline{\boldsymbol{z}}_{q}\right), \\
\boldsymbol{\lambda}_{i}\left(x_{0}, \overline{\boldsymbol{z}}_{i}\right)= & \boldsymbol{\phi}_{i}\left(x_{0}, \overline{\boldsymbol{z}}_{i}\right)-\frac{\partial \alpha_{i-1}\left(x_{0}, \overline{\boldsymbol{z}}_{i-1}\right)}{\partial x_{0}} \boldsymbol{\phi}_{0}\left(x_{0}\right)-\sum_{j=1}^{i-1} \frac{\partial \alpha_{i-1}\left(x_{0}, \overline{\boldsymbol{z}}_{i-1}\right)}{\partial z_{j}} \boldsymbol{\phi}_{j}\left(x_{0}, \overline{\boldsymbol{z}}_{j}\right),
\end{aligned}
$$


for $i=1, \ldots, n$ with $f_{n}\left(x_{0}, \boldsymbol{z}\right)=0$. Since $\phi_{0}(0)=0$ and $\phi_{i}(0,0)=0$ (due to $\left.u_{0}^{*}(0)=0\right)$ thanks to Assumption 1.1, we have

$$
\boldsymbol{\lambda}_{i}\left(x_{0}, \overline{\boldsymbol{z}}_{i}\right)=x_{0} \boldsymbol{\lambda}_{i 0}\left(x_{0}, \overline{\boldsymbol{z}}_{i}\right)+\sum_{j=1}^{i} e_{j} \boldsymbol{\lambda}_{i j}\left(x_{0}, \overline{\boldsymbol{z}}_{i}\right), i=1, \ldots, n,
$$

where $\boldsymbol{\lambda}_{i 0}(\bullet)$, and $\boldsymbol{\lambda}_{i j}(\bullet)$ are smooth functions of their arguments. We now design the virtual controls $\alpha_{i}\left(x_{0}, \overline{\boldsymbol{z}}_{i}\right)$ and the actual control $u_{1}\left(x_{0}, \boldsymbol{z}\right)$ simultaneously by considering the Lyapunov function candidate

$$
V_{1}(\boldsymbol{e})=\sum_{i=1}^{n} \gamma_{i}\left(\frac{e_{i}^{2 k}}{2 k}\right)
$$

where $\boldsymbol{e}=\operatorname{col}\left(e_{1}, \ldots, e_{n}\right)$, and $\gamma_{i}(\bullet)$ is a class $\mathcal{K}_{\infty}$ of $\bullet$ that has the same properties as $\gamma_{0}(\bullet)$ defined in (27), i.e.,

$$
\begin{aligned}
& 0<\gamma_{i}^{\prime} \leq a_{i}, \\
& \Delta_{i}\left(e_{i}\right)=\gamma_{i}^{\prime \prime} e_{i}^{2 k}+\gamma_{i}^{\prime}(2 k-1)>0,
\end{aligned}
$$

for all $e_{i} \in \mathbb{R}, \gamma_{i}^{\prime}=\partial \gamma_{i} / \partial\left(\frac{e_{i}^{2 k}}{2 k}\right), \gamma_{i}^{\prime \prime}=\partial^{2} \gamma_{i} / \partial\left(\frac{e_{i}^{2 k}}{2 k}\right)^{2}$, and $a_{i}$ is a positive constant. Using (62), the infinitesimal generator $\mathcal{L} V_{1}(\boldsymbol{e})$ can be calculated as

$$
\begin{aligned}
\mathcal{L} V_{1}(\boldsymbol{e})= & \frac{\partial V_{1}}{\partial e_{n}}\left(u_{1}+\Omega_{n}\left(x_{0}, \boldsymbol{z}\right)\right)+\sum_{i=1}^{n-1} \frac{\partial V_{1}}{\partial e_{i}}\left(e_{i+1}+\alpha_{i}\left(x_{0}, \overline{\boldsymbol{z}}_{i}\right)+\Omega_{i}\left(x_{0}, \overline{\boldsymbol{z}}_{i}\right)\right) \\
& +\frac{1}{2} \sum_{i=1}^{n} \operatorname{Tr}\left(\boldsymbol{\lambda}_{i}\left(x_{0}, \overline{\boldsymbol{z}}_{i}\right) \frac{\partial^{2} V_{1}}{\partial e_{i}^{2}} \boldsymbol{\lambda}_{i}^{T}\left(x_{0}, \overline{\boldsymbol{z}}_{i}\right)\right) \\
= & \gamma_{n}^{\prime} e_{n}^{2 k-1}\left(u_{1}+\Omega_{n}\left(x_{0}, \boldsymbol{z}\right)\right)+\sum_{i=1}^{n-1} \gamma_{i}^{\prime} e_{i}^{2 k-1}\left(\alpha_{i}\left(x_{0}, \overline{\boldsymbol{z}}_{i}\right)+\Omega_{i}\left(x_{0}, \overline{\boldsymbol{z}}_{i}\right)\right) \\
& +\sum_{i=1}^{n-1} \gamma_{i}^{\prime} e_{i}^{2 k-1} e_{i+1}+\frac{1}{2} \sum_{i=1}^{n} \Delta_{i}\left(e_{i}\right) e_{i}^{2 k-2}\left\|\boldsymbol{\lambda}_{i}\left(x_{0}, \overline{\boldsymbol{z}}_{i}\right)\right\|^{2},
\end{aligned}
$$

where $\Delta_{i}\left(e_{i}\right)$ is defined in (66). We now find the upper bounds of the last two terms in the right hand side of (67). Applying conditions (27) and (66) on $\gamma_{0}^{\prime}$ and $\gamma_{i}^{\prime}$, and the Young inequality yields

$$
\sum_{i=1}^{n-1} \gamma_{i}^{\prime} e_{i}^{2 k-1} e_{i+1} \leq \sum_{i=1}^{n} \gamma_{i}^{\prime} b_{i} e_{i}^{2 k}
$$

where $b_{i}$ is a positive constant. Using (64), conditions (27) and (66) on $\gamma_{0}^{\prime}$ and $\gamma_{i}^{\prime}$, and the Young inequality, it can be shown that

$$
\frac{1}{2} \Delta_{i}\left(e_{i}\right) e_{i}^{2 k-2}\left\|\boldsymbol{\lambda}_{i}\left(x_{0}, \overline{\boldsymbol{z}}_{i}\right)\right\|^{2} \leq \epsilon_{1 i 0} \gamma_{0}^{\prime} x_{0}^{2 k}+\sum_{j=1}^{i-1} \epsilon_{1 i j} \gamma_{j}^{\prime} e_{j}^{2 k}+\Phi_{i}\left(x_{0}, \overline{\boldsymbol{e}}_{i}\right) \gamma_{i}^{\prime} e_{i}^{2 k}, i=1, \ldots, n,
$$

where $\epsilon_{1 i j}$ with $i=1, . ., n$ and $j=0, . . i-1$ are nonnegative constants, and $\Phi_{i}\left(x_{0}, \bar{e}_{i}\right) \gamma_{i}^{\prime} e_{i}^{2 k}$ with $i=1, \ldots, n$ is a smooth function of $x_{0}$ and $\overline{\boldsymbol{e}}_{i}=\operatorname{col}\left(e_{1}, \ldots, e_{i}\right)$. It is noted that the function $\Phi_{i}\left(x_{0}, \overline{\boldsymbol{e}}_{i}\right)$ also depends on the constants $\epsilon_{1 i j}$. Summing the left and right hand sides of (69) yields

$$
\frac{1}{2} \sum_{i=1}^{n} \Delta_{i}\left(e_{i}\right) e_{i}^{2 k-2}\left\|\boldsymbol{\lambda}_{i}\left(x_{0}, \overline{\boldsymbol{z}}_{i}\right)\right\|^{2} \leq \epsilon_{10} \gamma_{0}^{\prime} x_{0}^{2 k}+\sum_{i=1}^{n-1} \epsilon_{1 i} \gamma_{i}^{\prime} e_{i}^{2 k}+\sum_{i=1}^{n} \gamma_{i}^{\prime} \Phi_{i}\left(x_{0}, \overline{\boldsymbol{e}}_{i}\right) e_{i}^{2 k},
$$

where

$$
\epsilon_{10}=\sum_{i=1}^{n} \epsilon_{1 i 0}, \quad \epsilon_{1 i}=\sum_{j=i+1}^{n} \epsilon_{1 j i} .
$$


We choose the constants $\epsilon_{1 i 0}$ with $i=1, \ldots, n$ such that $\epsilon_{10}$ is strictly less than $\epsilon$ defined in (33). Substituting (68) and (70) into (67) yields

$$
\mathcal{L} V_{1}(\boldsymbol{e}) \leq \sum_{i=1}^{n-1} \gamma_{i}^{\prime} e_{i}^{2 k-1}\left(\alpha_{i}+\Omega_{i}+q_{i}\left(x_{0}, \overline{\boldsymbol{e}}_{i}\right) e_{i}\right)+\gamma_{n}^{\prime} e_{n}^{2 k-1}\left(u_{1}+\Omega_{n}+q_{n}\left(x_{0}, \boldsymbol{e}\right) e_{n}\right)+\epsilon_{1} \gamma_{0}^{\prime} x_{0}^{2 k}
$$

where

$$
\begin{aligned}
& q_{i}\left(x_{0}, \overline{\boldsymbol{e}}_{i}\right)=b_{i}+\epsilon_{1 i}+\Phi_{i}\left(x_{0}, \overline{\boldsymbol{e}}_{i}\right), i=1, \ldots, n-1 \\
& q_{n}\left(x_{0}, \overline{\boldsymbol{e}}_{i}\right)=b_{n}+\Phi_{n}\left(x_{0}, \overline{\boldsymbol{e}}_{n}\right) .
\end{aligned}
$$

-Design of the inverse pre-optimal control $u_{1}^{\diamond}$ : Since $f_{i}(0,0)=0, \phi_{0}(0)=0$ and $\phi_{i}(0,0)=0$ (due to $\left.u_{0}^{*}(0)=0\right) \alpha_{i-1}\left(x_{0}, \overline{\boldsymbol{z}}_{i-1}\right)$ will be designed such that $\alpha_{i-1}(0,0)=0$ thanks to Assumption 1.1 , we have

$$
\Omega_{i}\left(x_{0}, \overline{\boldsymbol{z}}_{i}\right)=x_{0} \Omega_{i 0}\left(x_{0}, \overline{\boldsymbol{z}}_{i}\right)+\sum_{j=1}^{i} e_{j} \Omega_{i j}\left(x_{0}, \overline{\boldsymbol{z}}_{i}\right), i=1, \ldots, n,
$$

where $\Omega_{i 0}\left(x_{0}, \bar{z}_{i}\right)$ and $\Omega_{i j}\left(x_{0}, \overline{\boldsymbol{z}}_{i}\right)$ are smooth functions of $x_{0}$ and $\overline{\boldsymbol{z}}_{i}$. Using (74), conditions (27) and (66) on $\gamma_{0}^{\prime}$ and $\gamma_{i}^{\prime}$, and the Young inequality, it can be shown that

$$
\gamma_{i}^{\prime} e_{i}^{2 k-1} \Omega_{i} \leq \epsilon_{2 i 0} \gamma_{0}^{\prime} x_{0}^{2 k}+\sum_{j=1}^{i-1} \epsilon_{2 i j} \gamma_{j}^{\prime} e_{j}^{2 k}+\Psi_{i}\left(x_{0}, \overline{\boldsymbol{e}}_{i}\right) \gamma_{i}^{\prime} e_{i}^{2 k}, i=1, \ldots, n,
$$

where $\epsilon_{2 i j}$ with $j=0, . . i-1$ are nonnegative constants, and $\Psi_{i}\left(x_{0}, \overline{\boldsymbol{e}}_{i}\right) \gamma_{i}^{\prime} e_{i}^{2 k}$ is a smooth function of $x_{0}$ and $\overline{\boldsymbol{e}}_{i}=\operatorname{col}\left(e_{1}, \ldots, e_{i}\right)$. It is noted that the function $\Psi_{i}\left(x_{0}, \overline{\boldsymbol{e}}_{i}\right)$ also depends on the constants $\epsilon_{2 i j}$. Summing the left and right hand sides of (75) yields

$$
\sum_{i=1}^{n} \gamma_{i}^{\prime} e_{i}^{2 k-1} \Omega_{i} \leq \epsilon_{20} \gamma_{0}^{\prime} x_{0}^{2 k}+\sum_{i=1}^{n-1} \epsilon_{2 i} \gamma_{i}^{\prime} e_{i}^{2 k}+\sum_{i=1}^{n} \gamma_{i}^{\prime} \Psi_{i}\left(x_{0}, \overline{\boldsymbol{e}}_{i}\right) e_{i}^{2 k},
$$

where $\epsilon_{20}=\sum_{i=1}^{n} \epsilon_{2 i 0}$ and $\epsilon_{2 i}=\sum_{j=i+1}^{n} \epsilon_{2 j i}$. We choose the constants $\epsilon_{2 i 0}$ with $i=1, \ldots, n$ such that $\epsilon_{20}$ is strictly less than $\epsilon$ defined in (33). Substituting (68) and (76) into (72) yields

$$
\mathcal{L} V_{1}(\boldsymbol{e}) \leq \sum_{i=1}^{n-1} \gamma_{i}^{\prime} e_{i}^{2 k-1}\left(\alpha_{i}+p_{i}\left(x_{0}, \overline{\boldsymbol{e}}_{i}\right) e_{i}\right)+\gamma_{n}^{\prime} e_{n}^{2 k-1}\left(u_{1}+p_{n}\left(x_{0}, \boldsymbol{e}\right) e_{n}\right)+\left(\epsilon_{10}+\epsilon_{20}\right) \gamma_{0}^{\prime} x_{0}^{2 k},
$$

where

$$
\begin{aligned}
& p_{i}\left(x_{0}, \overline{\boldsymbol{e}}_{i}\right)=q_{i}\left(x_{0}, \overline{\boldsymbol{e}}_{i}\right)+\epsilon_{2 i}+\Psi_{i}\left(x_{0}, \overline{\boldsymbol{e}}_{i}\right), i=1, . ., n-1, \\
& p_{n}\left(x_{0}, \overline{\boldsymbol{e}}_{n}\right)=q_{n}\left(x_{0}, \overline{\boldsymbol{e}}_{n}\right)+\Psi_{n}\left(x_{0}, \overline{\boldsymbol{e}}_{n}\right) .
\end{aligned}
$$

From (77), we choose

$$
\begin{aligned}
& \alpha_{i}^{\diamond}=-\left(k_{i}+\sqrt{c_{i}+p_{i}^{2}\left(x_{0}, \overline{\boldsymbol{e}}_{i}\right)}\right) e_{i}, \quad i=1, \ldots, n-1 \\
& u_{1}^{\diamond}=-\left(k_{n}+\sqrt{c_{n}+p_{n}^{2}\left(x_{0}, \boldsymbol{e}\right)}\right) e_{n},
\end{aligned}
$$

where we have used the notations $\alpha_{i}^{\diamond}$ and $u_{1}^{\diamond}$ instead of $\alpha_{i}$ and $u_{1}$ to emphasize that $\alpha_{i}^{\diamond}$ and $u_{1}^{\diamond}$ are the inverse pre-optimal virtual and actual controls, and $c_{i}$ and $k_{i}, i=1, \ldots, n$ are positive constants.

Remark 5.1: From (72), Theorem 2.1 suggests that one would design the following control $u_{1}$ to asymptotically stabilize (62) in probability as $u_{1}=-k_{n} e_{n}-\Omega_{n}-q_{n}\left(x_{0}, \boldsymbol{e}\right) e_{n}$ with $\alpha_{i}=-k_{i} e_{i}-\Omega_{i}-$ $q_{i}\left(x_{0}, \overline{\boldsymbol{e}}_{i}\right) e_{i}, \quad i=1, \ldots, n-1$, where $k_{i}, i=1, \ldots n$, are positive constants. The above stabilizing control $u_{1}$ given cancels the term $\left(\Omega_{n}+q_{n}\left(x_{0}, \boldsymbol{e}\right) e_{n}\right)$ while the inverse pre-optimal control $u_{1}^{\diamond}$ in (79) dominates the term $p_{n}\left(x_{0}, \boldsymbol{e}\right) e_{n}$. This domination is necessary to make it possible to amend the inverse pre-optimal control so that optimality can be achieved as shown in Theorem 5.1.

The design of the inverse pre-optimal control $u_{1}^{\diamond}$ for the case $x_{0}\left(t_{0}\right) \neq 0$ has been completed. Substituting $u_{1}^{\diamond}$ given by (79) into (77) yields

$$
\left.\mathcal{L} V_{1}(\boldsymbol{e})\right|_{(79)} \leq-\sum_{i=1}^{n} k_{i} \gamma_{i}^{\prime} e_{i}^{2 k}+\left(\epsilon_{10}+\epsilon_{20}\right) \gamma_{0}^{\prime} x_{0}^{2 k}
$$


2) Design of the inverse optimal control $u_{1}^{*}$ : Applying Theorem 2.2 to (62) results in

$$
u_{1}^{*}=-\frac{\beta_{1}}{2} R_{1}^{-1} \gamma_{n}^{\prime} e_{n}^{2 k-1} \frac{\left(\sigma_{1}^{\star}\right)^{-1}\left(\left|R_{1}^{-1 / 2} \gamma_{n}^{\prime} e_{n}^{2 k-1}\right|\right)}{\left|R_{1}^{-1 / 2} \gamma_{n}^{\prime} e_{n}^{2 k-1}\right|},
$$

where $\beta_{1} \geq 2, \sigma_{1}$ is a class $\mathcal{K}_{\infty}$ function, $\sigma_{1}^{\star}(\bullet)=\frac{d \sigma_{1}}{d \bullet}$, the notation $\left(\sigma_{1}^{\star}\right)^{-1}(\bullet)$ denotes the inverse function of $\sigma_{1}^{\star}$, and since $u_{1}^{*}$ is scalar, $R_{1}(\boldsymbol{e})$ is sought to be a positive definite function of $\boldsymbol{e}$. The class $\mathcal{K}_{\infty}$ function $\sigma_{1}$ is chosen by the designer. We now determine $R_{1}\left(x_{0}, \boldsymbol{e}\right)$. By Theorem 2.2, the control $u_{1}^{\diamond}$, which stabilizes (62), should be of the form (20), i.e.,

$$
u_{1}^{\diamond}=-R_{1}^{-1} \gamma_{n}^{\prime} e_{n}^{2 k-1} \frac{\ell \sigma_{1}\left(\left|R_{1}^{-1 / 2} \gamma_{n}^{\prime} e_{n}^{2 k-1}\right|\right)}{\left|R_{1}^{-1 / 2} \gamma_{n}^{\prime} e_{n}^{2 k-1}\right|^{2}} .
$$

The positive definite function $R_{1}\left(x_{0}, \boldsymbol{e}\right)$ is to be determined so that the inverse pre-optimal control $u_{1}^{\diamond}$ given in (79) is the same with the one given in (82). As such, comparing (82) with (79) results in the fact that $R_{1}\left(x_{0}, \boldsymbol{e}\right)$ needs to satisfy the following equation

$$
R_{1}^{-1} \gamma_{n}^{\prime} e_{n}^{2 k-1} \frac{\ell \sigma_{1}\left(\left|R_{1}^{-1 / 2} \gamma_{n}^{\prime} e_{n}^{2 k-1}\right|\right)}{\left|R_{1}^{-1 / 2} \gamma_{n}^{\prime} e_{n}^{2 k-1}\right|^{2}}=\left(k_{n}+\sqrt{1+p_{n}^{2}\left(x_{0}, \boldsymbol{e}\right)}\right) e_{n} .
$$

Using the same technique in Subsection III-B1 to solve (83) for $R_{1}\left(x_{0}, \boldsymbol{e}\right)$, we find

$$
R_{1}\left(x_{0}, \boldsymbol{e}\right)=\left[\frac{\left|\gamma_{n}^{\prime} e_{n}^{2 k-1}\right|}{\left(\ell \sigma_{1}\right)^{-1}\left(\gamma_{n}^{\prime} e_{n}^{2 k}\left(k_{n}+\sqrt{c_{n}+p_{n}^{2}\left(x_{0}, \boldsymbol{e}\right)}\right)\right)}\right]^{2},
$$

which is continuous away from the origin and positive definite for all $x_{0} \in \mathbb{R}$ and $e \in \mathbb{R}^{n}$. Again, using technique in Subsection III-B1 we can write the inverse optimal control $u_{1}^{*}$ from (81) as

$$
u_{1}^{*}=-\frac{\beta_{1}}{2}\left[k_{n}+\sqrt{c_{n}+p_{n}^{2}\left(x_{0}, \boldsymbol{e}\right)}+R_{1}^{-1} \gamma_{n}^{\prime} e_{n}^{2 k-2} \frac{\sigma_{1}\left(\left(\sigma_{1}^{\star}\right)^{-1}\left(\left|R_{1}^{-1 / 2} \gamma_{n}^{\prime} e_{n}^{2 k-1}\right|\right)\right)}{\left|R_{1}^{-1 / 2} \gamma_{n}^{\prime} e_{n}^{2 k-1}\right|^{2}}\right] e_{n} .
$$

Substituting (85) and $\alpha_{i}$ given by (79) into (77) yields

$$
\left.\mathcal{L} V_{1}(\boldsymbol{e})\right|_{(85)} \leq-\left[\sum_{i=1}^{n-1} k_{i} \gamma_{i}^{\prime} e_{i}^{2 k}+\frac{\beta_{1} k_{n}}{2} \gamma_{n}^{\prime} e_{n}^{2 k}\right]+\left(\epsilon_{10}+\epsilon_{20}\right) \gamma_{0}^{\prime} x_{0}^{2 k},
$$

where we have used the fact that $\beta_{1} \geq 2$ and $k \geq 2$.

B. Case $x_{0}\left(t_{0}\right)=0$

Since the control $u_{0}^{*}$ is given in Procedure 3.2, the design of the stabilizing control $u_{1}$, the inverse preoptimal control $u_{1}^{\diamond}$, and the optimal control $u_{1}^{*}$ is slightly different from the design for the case $x_{0}\left(t_{0}\right) \neq 0$ when $t \leq t_{s}^{*}$. We only present the differences. When $t \leq t_{s}^{*}$, we have from Procedure 3.2 that $u_{0}^{*}(0)=\eta_{0}$, see (47). As a result, we cannot write $\boldsymbol{\lambda}_{i}\left(x_{0}, \overline{\boldsymbol{z}}_{i}\right)$ and $\Omega_{i}\left(x_{0}, \overline{\boldsymbol{z}}_{i}\right)$ as in (64) and (74), respectively, under Assumption 1.1 in general (an example is the case when the function $\boldsymbol{\varphi}_{i}\left(x_{0}, u_{0}, \boldsymbol{x}_{i}\right)$ contains a polynomial function of $u_{0}$ ). This means that we cannot obtain the bounds in (69) and (75) (or (70) and (76)). As such, we need to re-examine equations (64) and (74), and thus the inequalities (69) and (75).

1) If $t \leq t_{s}^{*}$ : Using $u_{0}^{*}(0)=\eta_{0}$ and Assumption 1.1, we can write $\boldsymbol{\lambda}_{i}\left(x_{0}, \overline{\boldsymbol{z}}_{i}\right)$ and $\Omega_{i}\left(x_{0}, \overline{\boldsymbol{z}}_{i}\right)$ as

$$
\begin{aligned}
& \boldsymbol{\lambda}_{i}\left(x_{0}, \overline{\boldsymbol{z}}_{i}\right)=x_{0} \overline{\boldsymbol{\lambda}}_{i 0}\left(x_{0}, \overline{\boldsymbol{z}}_{i}\right)+\sum_{j=1}^{i} e_{j} \overline{\boldsymbol{\lambda}}_{i j}\left(x_{0}, \overline{\boldsymbol{z}}_{i}\right)+\overline{\boldsymbol{\tau}}_{i 0}\left(x_{0}, \overline{\boldsymbol{z}}_{i}\right), \\
& \Omega_{i}\left(x_{0}, \overline{\boldsymbol{z}}_{i}\right)=x_{0} \bar{\Omega}_{i 0}\left(x_{0}, \overline{\boldsymbol{z}}_{i}\right)+\sum_{j=1}^{i} e_{j} \bar{\Omega}_{i j}\left(x_{0}, \overline{\boldsymbol{z}}_{i}\right)+\bar{\Theta}_{i 0}\left(x_{0}, \overline{\boldsymbol{z}}_{i}\right),
\end{aligned}
$$


where $\overline{\boldsymbol{\lambda}}_{i 0}\left(x_{0}, \overline{\boldsymbol{z}}_{i}\right), \overline{\boldsymbol{\lambda}}_{i j}\left(x_{0}, \overline{\boldsymbol{z}}_{i}\right), \overline{\boldsymbol{\tau}}_{i 0}\left(x_{0}, \overline{\boldsymbol{z}}_{i}\right), \bar{\Omega}_{i 0}\left(x_{0}, \overline{\boldsymbol{z}}_{i}\right), \bar{\Omega}_{i j}\left(x_{0}, \overline{\boldsymbol{z}}_{i}\right)$, and $\bar{\Theta}_{i 0}\left(x_{0}, \overline{\boldsymbol{z}}_{i}\right)$ are smooth functions of $x_{0}$ and $\overline{\boldsymbol{z}}_{i}$. It is noted that $\overline{\boldsymbol{\tau}}_{i 0}\left(x_{0}, \overline{\boldsymbol{z}}_{i}\right)$ and $\bar{\Theta}_{i 0}\left(x_{0}, \overline{\boldsymbol{z}}_{i}\right)$ depend on the constant $\eta_{0}$. Using the first equation of (87), conditions (27) and (66) on $\gamma_{0}^{\prime}$ and $\gamma_{i}^{\prime}$, and the Young inequality, it can be shown that

$$
\frac{1}{2} \Delta_{i}\left(e_{i}\right) e_{i}^{2 k-2}\left\|\boldsymbol{\lambda}_{i}\left(x_{0}, \overline{\boldsymbol{z}}_{i}\right)\right\|^{2} \leq \epsilon_{1 i 0} \gamma_{0}^{\prime} x_{0}^{2 k}+\sum_{j=1}^{i-1} \epsilon_{1 i j} \gamma_{j}^{\prime} e_{j}^{2 k}+\bar{\Phi}_{i}\left(x_{0}, \overline{\boldsymbol{e}}_{i}\right) \gamma_{i}^{\prime} e_{i}^{2 k}+\bar{\Phi}_{i 0}, i=1, \ldots, n,
$$

where $\bar{\Phi}_{i}\left(x_{0}, \overline{\boldsymbol{e}}_{i}\right)$ are smooth functions of $x_{0}$ and $\overline{\boldsymbol{e}}_{i}$, and $\bar{\Phi}_{i 0}$ are nonnegative constants depending on the constant $\eta_{0}$. Similarly, using the second equation of (87), conditions (27) and (66) on $\gamma_{0}^{\prime}$ and $\gamma_{i}^{\prime}$, and the Young inequality, it can be shown that

$$
\gamma_{i}^{\prime} e_{i}^{2 k-1} \Omega_{i} \leq \epsilon_{2 i 0} \gamma_{0}^{\prime} x_{0}^{2 k}+\sum_{j=1}^{i-1} \epsilon_{2 i j} \gamma_{j}^{\prime} e_{j}^{2 k}+\bar{\Psi}_{i}\left(x_{0}, \overline{\boldsymbol{e}}_{i}\right) \gamma_{i}^{\prime} e_{i}^{2 k}+\bar{\Psi}_{i 0}, i=1, \ldots, n,
$$

where $\bar{\Psi}_{i}\left(x_{0}, \overline{\boldsymbol{e}}_{i}\right)$ is a smooth function of $x_{0}$ and $\overline{\boldsymbol{e}}_{i}$, and $\bar{\Psi}_{i 0}$ is a nonnegative constant depending on the constant $\eta_{0}$. Summing the left and right hand sides of (88) and (89) yields

$$
\begin{aligned}
& \frac{1}{2} \sum_{i=1}^{n} \Delta_{i}\left(e_{i}\right) e_{i}^{2 k-2}\left\|\boldsymbol{\lambda}_{i}\left(x_{0}, \overline{\boldsymbol{z}}_{i}\right)\right\|^{2} \leq \epsilon_{10} \gamma_{0}^{\prime} x_{0}^{2 k}+\sum_{i=1}^{n-1} \epsilon_{1 i} \gamma_{i}^{\prime} e_{i}^{2 k}+\sum_{i=1}^{n} \gamma_{i}^{\prime} \bar{\Phi}_{i}\left(x_{0}, \overline{\boldsymbol{e}}_{i}\right) e_{i}^{2 k}+\bar{\Phi}_{0}, \\
& \sum_{i=1}^{n} \gamma_{i}^{\prime} e_{i}^{2 k-1} \Omega_{i} \leq \epsilon_{20} \gamma_{0}^{\prime} x_{0}^{2 k}+\sum_{i=1}^{n-1} \epsilon_{2 i} \gamma_{i}^{\prime} e_{i}^{2 k}+\sum_{i=1}^{n} \gamma_{i}^{\prime} \bar{\Psi}_{i}\left(x_{0}, \overline{\boldsymbol{e}}_{i}\right) e_{i}^{2 k}+\bar{\Psi}_{0},
\end{aligned}
$$

where $\bar{\Phi}_{0}=\sum_{i=1}^{n} \bar{\Phi}_{i 0}$ and $\bar{\Psi}_{0}=\sum_{i=1}^{n} \bar{\Psi}_{i 0}$. Substituting (68) and the first inequality of (90) into (67) yields

$$
\mathcal{L} V_{1}(\boldsymbol{e}) \leq \sum_{i=1}^{n-1} \gamma_{i}^{\prime} e_{i}^{2 k-1}\left(\alpha_{i}+\Omega_{i}+\bar{q}_{i}\left(x_{0}, \overline{\boldsymbol{e}}_{i}\right) e_{i}\right)+\gamma_{n}^{\prime} e_{n}^{2 k-1}\left(u_{1}+\Omega_{n}+\bar{q}_{n}\left(x_{0}, \boldsymbol{e}\right) e_{n}\right)+\epsilon_{10} \gamma_{0}^{\prime} x_{0}^{2 k}+\bar{\Phi}_{0},
$$

where

$$
\begin{aligned}
& \bar{q}_{i}\left(x_{0}, \overline{\boldsymbol{e}}_{i}\right)=b_{i}+\epsilon_{1 i}+\bar{\Phi}_{i}\left(x_{0}, \overline{\boldsymbol{e}}_{i}\right), i=1, \ldots, n-1, \\
& \bar{q}_{n}\left(x_{0}, \overline{\boldsymbol{e}}_{n}\right)=b_{n}+\bar{\Phi}_{n}\left(x_{0}, \overline{\boldsymbol{e}}_{n}\right) .
\end{aligned}
$$

with $b_{i}$ being defined just below (68). From (91), one would design a stabilizing control $u_{1}$ as $u_{1}=$ $-k_{n} e_{n}-\Omega_{n}-\bar{q}_{n}\left(x_{0}, \boldsymbol{e}\right) e_{n}$ with $\alpha_{i}=-k_{i} e_{i}-\Omega_{i}-\bar{q}_{i}\left(x_{0}, \overline{\boldsymbol{e}}_{i}\right) e_{i}, \quad i=1, \ldots, n-1$. However, this stabilizing control cannot be amended to become an (inverse) optimal control as noted in Remark 5.1.

-Design of the inverse pre-optimal control $u_{1}^{\diamond}$ : Substituting the second inequality of (90) into (91) yields

$$
\mathcal{L} V_{1}(\boldsymbol{e}) \leq \sum_{i=1}^{n-1} \gamma_{i}^{\prime} e_{i}^{2 k-1}\left(\alpha_{i}+\bar{p}_{i}\left(x_{0}, \overline{\boldsymbol{e}}_{i}\right) e_{i}\right)+\gamma_{n}^{\prime} e_{n}^{2 k-1}\left(u_{1}+\bar{p}_{n}\left(x_{0}, \boldsymbol{e}\right) e_{n}\right)+\left(\epsilon_{10}+\epsilon_{20}\right) \gamma_{0}^{\prime} x_{0}^{2 k}+\bar{\Phi}_{0}+\bar{\Psi}_{0},
$$

where

$$
\begin{aligned}
& \bar{p}_{i}\left(x_{0}, \overline{\boldsymbol{e}}_{i}\right)=\bar{q}_{i}\left(x_{0}, \overline{\boldsymbol{e}}_{i}\right)+\epsilon_{2 i}+\bar{\Psi}_{i}\left(x_{0}, \overline{\boldsymbol{e}}_{i}\right), i=1, \ldots, n-1, \\
& \bar{p}_{n}\left(x_{0}, \overline{\boldsymbol{e}}_{n}\right)=\bar{q}_{n}\left(x_{0}, \overline{\boldsymbol{e}}_{n}\right)+\bar{\Psi}_{n}\left(x_{0}, \overline{\boldsymbol{e}}_{n}\right) .
\end{aligned}
$$

From (93), we choose

$$
\begin{aligned}
& \alpha_{i}^{\diamond}=-\left(k_{i}+\sqrt{c_{i}+\bar{p}_{i}^{2}\left(x_{0}, \overline{\boldsymbol{e}}_{i}\right)}\right) e_{i}, \quad i=1, \ldots, n-1 \\
& u_{1}^{\diamond}=-\left(k_{n}+\sqrt{c_{n}+\bar{p}_{n}^{2}\left(x_{0}, \boldsymbol{e}\right)}\right) e_{n} .
\end{aligned}
$$


Substituting (95) into (93) yields

$$
\left.\mathcal{L} V_{1}(\boldsymbol{e})\right|_{(95)} \leq-\sum_{i=1}^{n} k_{i} \gamma_{i}^{\prime} e_{i}^{2 k}+\left(\epsilon_{10}+\epsilon_{20}\right) \gamma_{0}^{\prime} x_{0}^{2 k}+\bar{\Phi}_{0}+\bar{\Psi}_{0}
$$

-Design of the inverse optimal control $u_{1}^{*}$ : Using the inverse pre-optimal control $u_{1}^{\diamond}$ and the same technique in Subsection V-A2, we find

$$
u_{1}^{*}=-\frac{\beta_{1}}{2}\left[k_{n}+\sqrt{c_{n}+\bar{p}_{n}^{2}\left(x_{0}, \boldsymbol{e}\right)}+R_{1}^{-1} \gamma_{n}^{\prime} e_{n}^{2 k-2} \frac{\sigma_{1}\left(\left(\sigma_{1}^{\star}\right)^{-1}\left(\left|R_{1}^{-1 / 2} \gamma_{n}^{\prime} e_{n}^{2 k-1}\right|\right)\right)}{\left|R_{1}^{-1 / 2} \gamma_{n}^{\prime} e_{n}^{2 k-1}\right|^{2}}\right] e_{n},
$$

where

$$
R_{1}\left(x_{0}, \boldsymbol{e}\right)=\left[\frac{\left|\gamma_{n}^{\prime} e_{n}^{2 k-1}\right|}{\left(\ell \sigma_{1}\right)^{-1}\left(\gamma_{n}^{\prime} e_{n}^{2 k}\left(k_{n}+\sqrt{c_{n}+\bar{p}_{n}^{2}\left(x_{0}, \boldsymbol{e}\right)}\right)\right)}\right]^{2} .
$$

Substituting (97) and $\alpha_{i}$ given by (95) into (93) yields

$$
\left.\mathcal{L} V_{1}(\boldsymbol{e})\right|_{(97)} \leq-\left[\sum_{i=1}^{n-1} k_{i} \gamma_{i}^{\prime} e_{i}^{2 k}+\frac{\beta_{1} k_{n}}{2} \gamma_{n}^{\prime} e_{n}^{2 k}\right]+\left(\epsilon_{10}+\epsilon_{20}\right) \gamma_{0}^{\prime} x_{0}^{2 k}+\bar{\Phi}_{0}+\bar{\Psi}_{0}
$$

2) If $t>t_{s}^{*}$ : The stabilizing control $u_{1}$, the inverse pre-optimal control $u_{1}^{\diamond}$, and the optimal control $u_{1}^{*}$ are the same as those for the case $x_{0}\left(t_{0}\right) \neq 0$.

Note that all the virtual controls $\alpha_{i}^{\diamond}$ and the controls $u_{1}^{\diamond}$ and $u_{1}^{*}$ are discontinuous at $t=t_{s}^{*}$. Due to strong nonlinear functions $\varphi_{i}$ in (1), if one applies a control input $u_{1}^{\diamond}=$ constant or $u_{1}^{*}=$ constant for $t_{0} \leq t \leq t_{s}^{*}$ as proposed in [13], [7], the solution of the $\boldsymbol{x}$-subsystem may blow up before the control $u_{0}^{*}$ is switched.

We now present the second result in the following theorem.

Theorem 5.1: Under Assumption 1.1, for any initial values $x_{0}\left(t_{0}\right) \in \mathbb{R}$ and $x_{i}\left(t_{0}\right) \in \mathbb{R}$ with $i=1, \ldots, n$, the following results hold:

1) Case $x_{0}\left(t_{0}\right) \neq 0$ or case $x_{0}\left(t_{0}\right)=0$ when $t>t_{s}^{*}$ : With the inverse optimal control $u_{0}^{*}$ given by (45) or (48), the inverse pre-optimal control $u_{1}^{\diamond}$ given by (79), ensures that the solution $\boldsymbol{x}(t)=\operatorname{col}\left(x_{1}(t), \ldots, x_{n}(t)\right)$ of the $\boldsymbol{x}$-subsystem exists uniquely and asymptotically converges to zero in probability.

Under an additional condition on the class $\mathcal{K}_{\infty}$ function $\sigma_{1}$ that $\sigma_{1}^{\star}$ is also a class $\mathcal{K}_{\infty}$ function, the inverse optimal control $u_{1}^{*}$ given by (85) guarantees existence, uniqueness, and global asymptotic convergence to zero in probability of the solution $\boldsymbol{x}(t)$. Moreover, the following cost functional is minimized:

$$
J_{1}\left(u_{1}\right)=\mathbb{E}\left\{\int_{t_{0}^{*}}^{\infty}\left[l_{1}\left(x_{0}, \boldsymbol{x}\right)+\beta_{1}^{2} \sigma_{1}\left(\frac{2}{\beta_{1}}\left|R_{1}^{1 / 2} u_{1}\right|\right)\right] d t\right\},
$$

where $t_{0}^{*}=\max \left(t_{0}, t_{s}^{*}\right)$ and

$$
\left.l_{1}\left(x_{0}, \boldsymbol{x}\right)=2 \beta_{1}\left[\ell \sigma_{1}\left(\left|\gamma_{n}^{\prime} e_{n}^{2 k-1} R_{n}^{-1 / 2}\right|\right)-\frac{1}{2} \Delta_{n}\left(e_{n}\right)\left\|\boldsymbol{\varphi}_{n}\left(x_{0}, \boldsymbol{e}\right)\right\|^{2}\right)\right]+\beta_{1}\left(\beta_{1}-2\right) \ell \sigma_{1}\left(\left|\gamma_{n}^{\prime} e_{n}^{2 k-1} R_{1}^{-1 / 2}\right|\right),
$$

with $\Delta_{n}\left(e_{n}\right)$ being given by (66).

2) Case $x_{0}\left(t_{0}\right)=0$ when $t \leq t_{s}^{*}$ : With the inverse optimal control $u_{0}^{*}$ given by (47), the inverse preoptimal control $u_{1}^{\diamond}$ given by (95) or the inverse optimal control $u_{1}^{*}$ given by (97) guarantees existence and uniqueness of the solution $\boldsymbol{x}(t)$ of the $\boldsymbol{x}$-subsystem.

Proof. See Appendix D. 


\section{Example 1.1 (cont'd)}

We continue Example 1.1 to illustrate the control design developed in this section. From (61), we have

$$
e_{1}=z_{1}, \quad e_{2}=z_{2}-\alpha_{1}\left(x_{0}, z_{1}\right) .
$$

Applying (62) results in the error dynamics

$$
\begin{aligned}
& d e_{1}=\left(e_{2}+\alpha_{1}\left(x_{0}, z_{1}\right)+\Omega_{1}\left(x_{0}, z_{1}\right)\right) d t+\lambda_{1}\left(x_{0}, z_{1}\right) d w, \\
& d e_{2}=\left(u_{1}+\Omega_{2}\left(x_{0}, z_{1}, z_{2}\right)\right) d t+\lambda_{2}\left(x_{0}, z_{1}, z_{2}\right) d w,
\end{aligned}
$$

where from (63) we have

$$
\begin{aligned}
& \Omega_{1}\left(x_{0}, z_{1}\right)=f_{1}\left(x_{0}, z_{1}\right), \lambda_{1}\left(x_{0}, z_{1}\right)=0, \\
& \Omega_{2}\left(x_{0}, z_{1}, z_{2}\right)=-\frac{\partial \alpha_{1}}{\partial x_{0}} u_{0}^{*}\left(x_{0}\right)-\frac{\partial \alpha_{1}^{\diamond}}{\partial z_{1}}\left(z_{2}+f_{1}\right), \lambda_{2}\left(x_{0}, z_{1}, z_{2}\right)=\phi_{2}\left(x_{0}, z_{1}, z_{2}\right),
\end{aligned}
$$

with $u_{0}^{*}\left(x_{0}\right)$ being given in (54) for the case $x_{0}\left(t_{0}\right) \neq 0$, and (55) and (56) for the case $x_{0}\left(t_{0}\right)=0$, and $f_{1}\left(x_{0}, z_{1}\right)$ and $\phi_{2}\left(x_{0}, z_{1}, z_{2}\right)$ being given in (60).

1) Design of the virtual control $\alpha_{1}^{\diamond}$ : Applying (64) and (74) to (104) with $\phi_{1}\left(x_{0}, z_{1}\right)$ and $f_{1}\left(x_{0}, z_{1}\right)$ being given in (60), and $\varphi_{0}\left(x_{0}\right)=0$ and $\varphi_{1}\left(x_{0}, x_{1}\right)=0$, see Subsection III-C, results in the following factors of $\lambda_{1}\left(x_{0}, z_{1}\right)$ and $\Omega_{1}\left(x_{0}, z_{1}\right)$ :

$$
\lambda_{10}=0, \lambda_{11}=0, \Omega_{10}=0, \Omega_{11}=-\frac{1}{u_{0}^{*}} \frac{\partial u_{0}^{*}}{\partial x_{0}} u_{0}^{*} .
$$

We choose $\gamma_{1}(\chi)=\chi$ and $\gamma_{2}(\chi)=\chi$. This choice gives $\gamma_{1}^{\prime}=\gamma_{2}^{\prime}=1, \gamma_{1}^{\prime \prime}=\gamma_{2}^{\prime \prime}=0$, and $\Delta_{1}=\Delta_{2}=2 k-1$. Calculating the following bounds according to (68), (69) and (75) using (105) results in

$$
\begin{aligned}
& b_{1}=\frac{2 k-1}{2 k} \epsilon_{0}^{\frac{2 k-1}{2 k}}, b_{2}=\frac{1}{2 k \epsilon_{0}^{2 k}}, \\
& \epsilon_{110}=0, \Phi_{1}\left(x_{0}, z_{1}\right)=0, \epsilon_{210}=0, \Psi_{1}\left(x_{0}, z_{1}\right)=\Omega_{11}, \\
& \epsilon_{11}=\epsilon_{121}, \epsilon_{21}=\epsilon_{221} .
\end{aligned}
$$

With (106), we calculate $q_{1}\left(x_{0}, e_{1}\right)$ and $p_{1}\left(x_{0}, e_{1}\right)$ from (73) and (78), respectively, as follows:

$$
q_{1}\left(x_{0}, e_{1}\right)=b_{1}+\epsilon_{11}+\Phi_{1}, p_{1}\left(x_{0}, e_{1}\right)=q_{1}+\epsilon_{21}+\Psi_{1} .
$$

The inverse pre-optimal virtual control $\alpha_{1}^{\diamond}$ is designed based on (79) as follows:

$$
\alpha_{1}^{\diamond}\left(x_{0}, e_{1}\right)=-\left(k_{1}+\sqrt{c_{1}+p_{1}^{2}\left(x_{0}, e_{1}\right)}\right) e_{1} .
$$

2) Design of the control $u_{1}^{\diamond}$ and $u_{1}^{*}$ : Applying (64) and (74) to (104) with $\phi_{1}\left(x_{0}, z_{1}\right), \phi_{2}\left(x_{0}, z_{1}, z_{2}\right)$, and $f_{1}\left(x_{0}, z_{1}\right)$ being given in (60), and and $\varphi_{0}\left(x_{0}\right)=0, \varphi_{1}\left(x_{0}, x_{1}\right)=0$, and $\varphi_{2}\left(x_{0}, u_{0}, x_{1}, x_{2}\right)=x_{0}^{2}+x_{1}^{2}+x_{2}^{2}$, see Subsection III-C, and $\alpha_{1}^{\diamond}$ being given in (108) results in the following factors of $\lambda_{2}\left(x_{0}, z_{1}, z_{2}\right)$ and $\Omega_{2}\left(x_{0}, z_{1}, z_{2}\right)$ :

$$
\begin{aligned}
& \lambda_{20}\left(x_{0}, z_{1}, z_{2}\right)=x_{0}, \lambda_{21}\left(x_{0}, z_{1}, z_{2}\right)=u_{0}^{* 2} e_{1}+\alpha_{11}^{\diamond} e_{1}, \lambda_{22}\left(x_{0}, z_{1}, z_{2}\right)=e_{2}+2 \alpha_{11}^{\diamond} e_{1}, \\
& \Omega_{20}\left(x_{0}, z_{1}, z_{2}\right)=0, \Omega_{21}\left(x_{0}, z_{1}, z_{2}\right)=-\frac{\partial \alpha_{11}^{\diamond}}{\partial x_{0}} u_{0}^{*}\left(x_{0}\right)-\frac{\partial \alpha_{1}^{\diamond}}{\partial z_{1}}\left(\alpha_{11}^{\diamond}+\Omega_{11}\right), \Omega_{22}\left(x_{0}, z_{1}, z_{2}\right)=-\frac{\partial \alpha_{1}^{\diamond}}{\partial z_{1}},
\end{aligned}
$$

where $\alpha_{11}^{\diamond}\left(x_{0}, e_{1}\right)=-\left(k_{1}+\sqrt{c_{1}+p_{1}^{2}\left(x_{0}, e_{1}\right)}\right)$. With (109), we calculate the following bounds as in (69) and (75) to obtain

$$
\begin{aligned}
& \epsilon_{120}=\frac{1}{k \epsilon_{0}^{k}}, \epsilon_{121}=\frac{1}{k \epsilon_{0}^{k}}, \Phi_{2}\left(x_{0}, e_{1}, e_{2}\right)=\frac{k-1}{k}\left(\epsilon_{0} \frac{3 \Delta_{2}}{2}\right)^{\frac{k}{k-1}}\left(\lambda_{20}^{\frac{2 k}{k-1}}+\lambda_{21}^{\frac{2 k}{k-1}}\right)+\frac{3 \Delta_{2}}{2} \lambda_{22}^{2}, \\
& \epsilon_{220}=0, \epsilon_{221}=\frac{1}{2 k \epsilon_{0}^{2 k}}, \Psi_{2}\left(x_{0}, e_{1}, e_{2}\right)=\frac{2 k-1}{2 k}\left(\epsilon_{0} \Omega_{21}\right)^{\frac{2 k}{2 k-1}}+\Omega_{22} .
\end{aligned}
$$


With (110), we calculate $q_{2}\left(x_{0}, e_{1}, e_{2}\right)$ and $p_{2}\left(x_{0}, e_{1}, e_{2}\right)$ from (73) and (78), respectively, as follows:

$$
q_{2}\left(x_{0}, e_{1}, e_{2}\right)=b_{2}+\Phi_{2}, p_{2}\left(x_{0}, e_{1}, e_{2}\right)=q_{2}+\Psi_{2} .
$$

The inverse pre-optimal control $u_{1}^{\diamond}$ is obtained from (79) as follows:

$$
u_{1}^{\diamond}=-\left(k_{2}+\sqrt{c_{2}+p_{2}^{2}\left(x_{0}, e_{1}, e_{2}\right)}\right) e_{2} .
$$

Now we choose $\sigma_{1}(\chi)=\frac{\chi^{4}}{4}$. Thus, we have $\sigma_{1}^{\star}(\chi)=\chi^{3},\left(\sigma_{1}^{\star}\right)^{-1}(\chi)=\chi^{1 / 3}, \ell \sigma_{1}(\chi)=\frac{3}{4} \chi^{4 / 3}$, and $\left(\ell \sigma_{1}\right)^{-1}(\chi)=\left(\frac{4}{3} \chi\right)^{3 / 4}$. The function $R_{1}\left(x_{0}, e_{1}, e_{2}\right)$ is obtained from (84) as

$$
R_{1}\left(x_{0}, e_{1}, e_{2}\right)=\frac{\left|e_{2}^{k-2}\right|}{\left(\frac{4}{3}\left(k_{2}+\sqrt{c_{2}+p_{2}^{2}\left(x_{0}, e_{1}, e_{2}\right)}\right)\right)^{3 / 2}} .
$$

Therefore, the inverse optimal control $u_{1}^{*}$ is obtained from (85) as follows:

$$
u_{1}^{*}=-\frac{2 \beta_{1}}{3}\left(k_{2}+\sqrt{c_{2}+p_{2}^{2}\left(x_{0}, e_{1}, e_{2}\right)}\right) e_{2} .
$$

The above controls $u_{1}^{\diamond}$ and $u_{1}^{*}$ are for both cases $x_{0}\left(t_{0}\right) \neq 0$ and $x_{0}\left(t_{0}\right)=0$ because $\lambda_{1}(0,0)=0$, $\lambda_{2}(0,0,0)=0, \Omega_{1}(0,0)=0$, and $\Omega_{2}(0,0,0)=0$ regardless $u_{0}^{*}$ given by $(54)$ or $(55)$ or $(56)$.
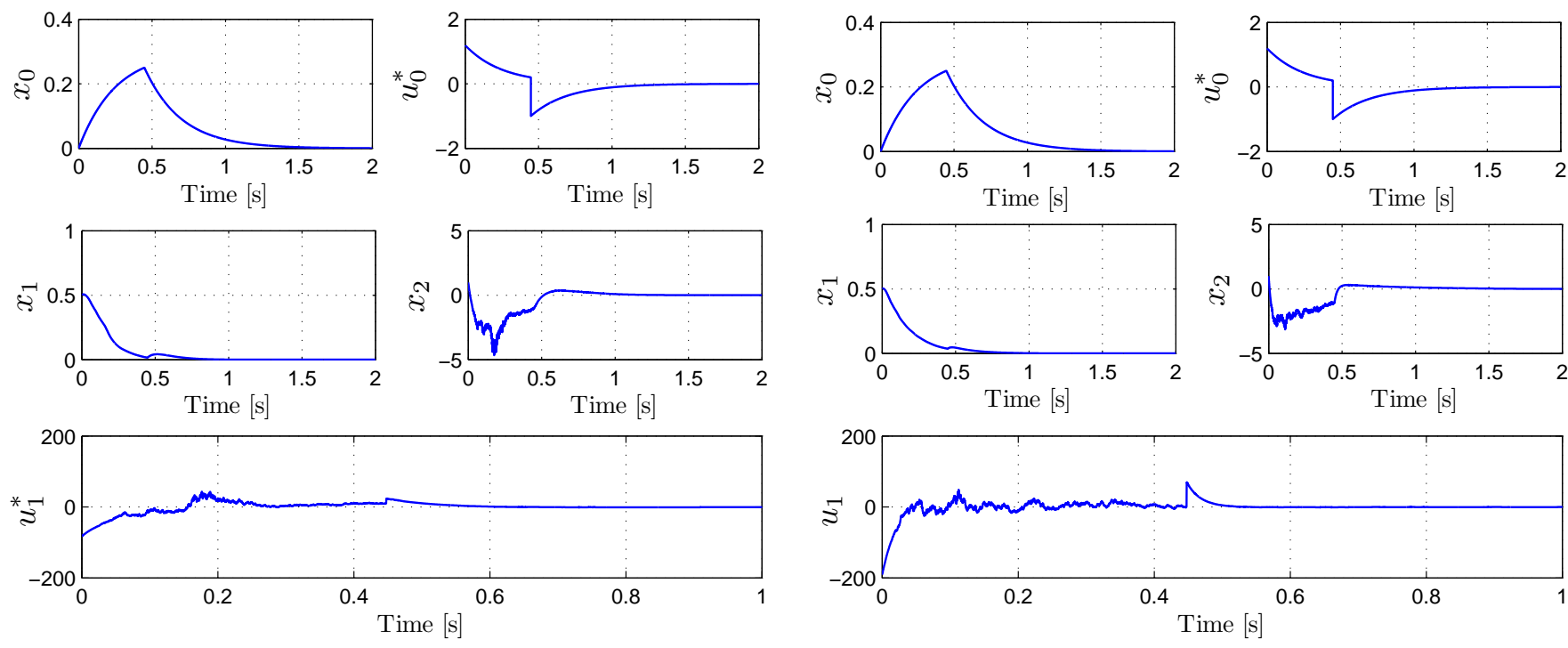

(a) Results with the proposed optimal control $u_{1}^{*}$

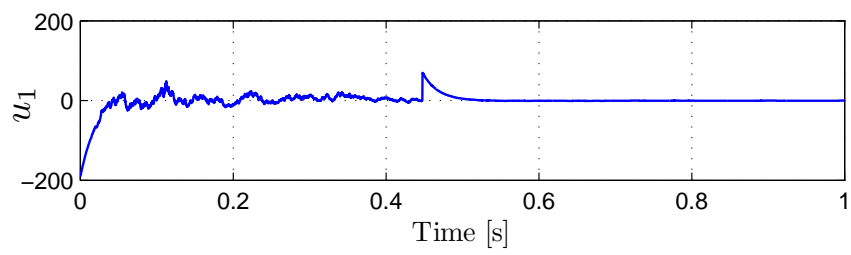

(b) Results with a stabilizing control $u_{1}$

Fig. 2: Simulation results with the proposed optimal control $u_{1}^{*}$ and a stabilizing control $u_{1}$.

In simulations, we choose $\epsilon_{0}=1, k=2, k_{0}=2, k_{1}=1, k_{2}=2, \beta_{0}=2$, and $\beta_{1}=2$. It is checked that $k_{0}$ satisfies the condition (33). We only provide simulation results for the case $x_{0}\left(t_{0}\right)=0$ since the results for the case $x_{0}\left(t_{0}\right) \neq 0$ are a part of those for the case $x_{0}\left(t_{0}\right)=0$ for $t \geq t_{s}^{*}$. Figure 2a presents the results with the initial conditions $\left(x_{0}(0), x_{1}(0), x_{2}(0)\right)=(0,0.5,1)$, where the switching parameters are chosen as $\delta_{0}=1$ and $\eta_{0}=1.2$. For a comparison, we also provide simulation results in Fig. $2 \mathrm{~b}$ with the following stabilizing control $u_{1}$ obtained from Remark 5.1:

$$
u_{1}=-k_{2} e_{2}-q_{2} e_{2}-\Omega_{2} e_{2},
$$

where $q_{2}$ and $\Omega_{2}$ are given (111) and (104), respectively. It is observed from Figs. 2a and 2b that although convergence of the states $x_{0}, x_{1}$, and $x_{2}$ to zero is similar for both controls $u_{1}^{*}$ given in (114) and $u_{1}$ given in (115), the difference is in the control effort. The magnitude of the stabilizing control $u_{1}$ $\left(\sup _{t \geq 0}\left|u_{1}(t)\right|=190.8\right)$ is more than double of the inverse optimal control $u_{1}^{*}\left(\sup _{t \geq 0}\left|u_{1}^{*}(t)\right|=82.72\right)$. 
This is a desired property of an inverse optimal control over a stabilizing one. Basically, the third term inside the square bracket in (85) or (97) multiplied by $-\frac{\beta_{1}}{2} e_{n}$ cancels destabilizing terms in $e_{n}$-dynamics when it is necessary otherwise strengthens stability. A stabilizer obtained from Remark 5.1 or (115) always cancels the destabilizing terms, see [33] (Sections 3.3-3.5) for more discussion on advantages of an inverse optimal control over a stabilizer for deterministic systems.

Since the first two equations of (5) do not contain noise, the states $x_{0}(t)$ and $x_{1}(t)$ are not affected by noise while the state $x_{2}(t)$ contains attenuating noise since the function $\varphi_{2}\left(x_{0}, x_{1}, x_{2}\right)$ vanishes when its arguments do. Also, all the controls and states are discontinuous when $\vartheta_{0}^{*}\left(x_{0}\right)=\delta_{0}$ (at $t_{s}^{*} \approx 0.446 \mathrm{~s}$ ).

\section{CONTROL DESIGN SUMMARIZATION}

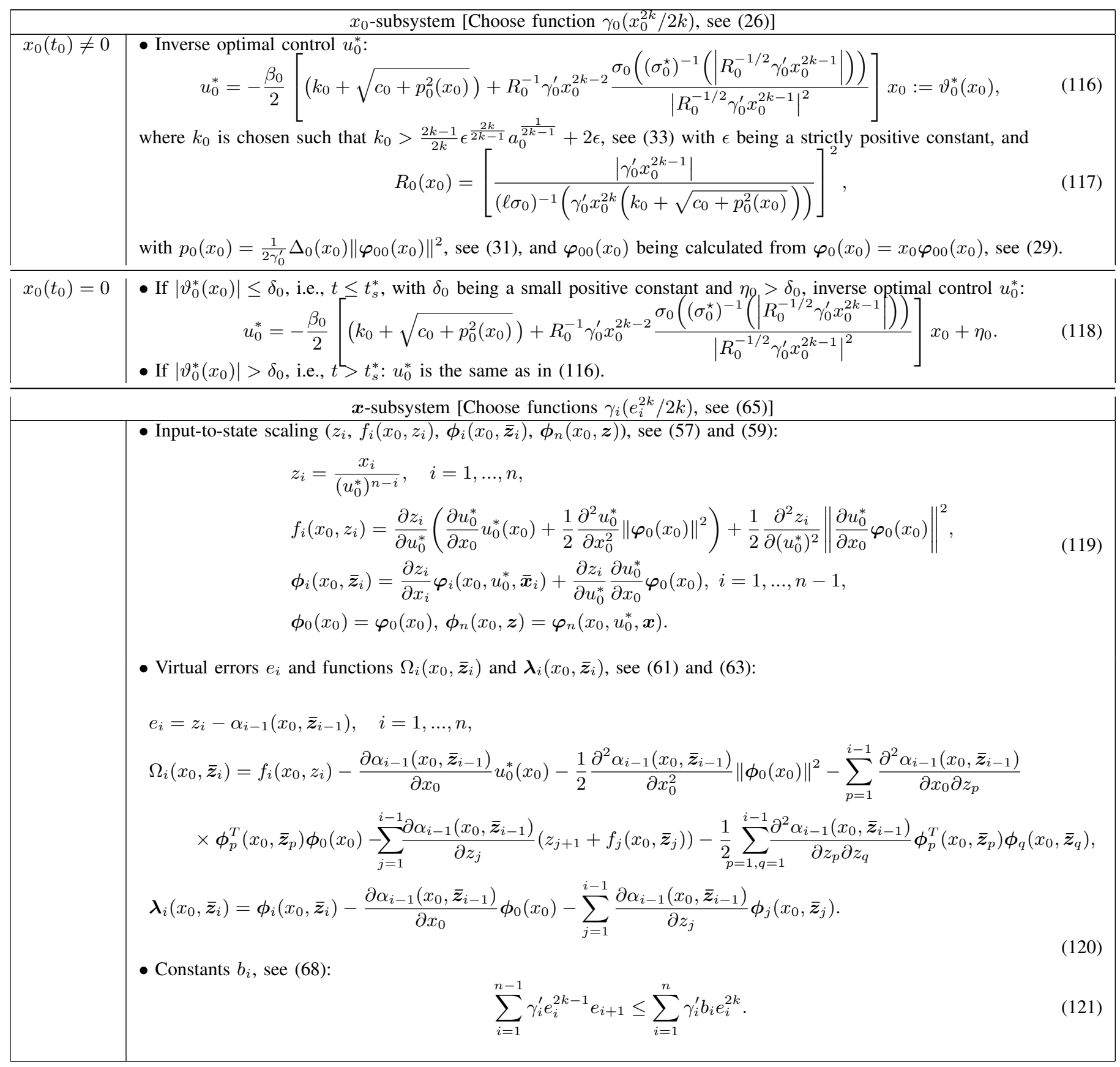




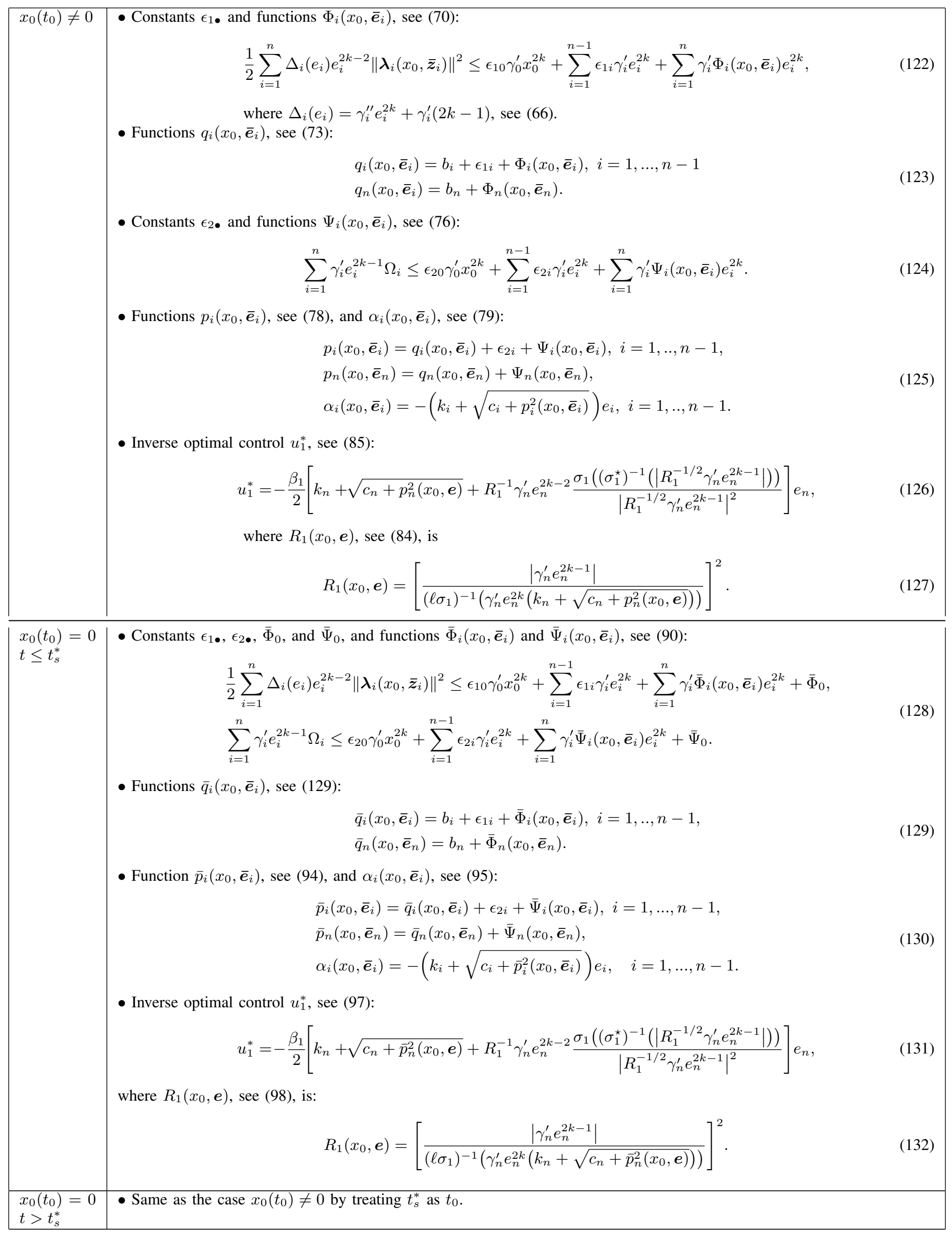


The proposed inverse optimal control design is summarized in Table I. From this table, it is seen that a nonexpert in stochastic theory can apply the proposed control design to the stochastic nonholonomic system (1) since it just requires several fundamental calculations such as partial differentiations and inverse functions. As such, one should start with the $x_{0}$-subsystem by designing the inverse optimal control $u_{0}^{*}$ as in (116) for the case $x_{0}\left(t_{0}\right) \neq 0$ or as in (118) for the case $x_{0}\left(t_{0}\right)=0$. For the $\boldsymbol{x}$-subsystem, one should follow the following steps for the case $x_{0}\left(t_{0}\right) \neq 0$ : 1) Perform input-to-state scaling to obtain the state $z_{i}$ and functions $\left(f_{i}\left(x_{0}, z_{i}\right), \boldsymbol{\phi}_{i}\left(x_{0}, \overline{\boldsymbol{z}}_{i}\right), \boldsymbol{\phi}_{n}\left(x_{0}, \boldsymbol{z}\right)\right)$ as in (119); 2) Calculate the virtual errors $e_{i}$ and functions $\Omega_{i}\left(x_{0}, \overline{\boldsymbol{z}}_{i}\right)$ and $\boldsymbol{\lambda}_{i}\left(x_{0}, \overline{\boldsymbol{z}}_{i}\right)$ as in (120). Note that this step requires $n$ sub-steps; 3$)$ Calculate the constants $b_{i}$ as in (121), $\epsilon_{1}$ • and functions $\Phi_{i}\left(x_{0}, \overline{\boldsymbol{e}}_{i}\right)$ as in (122), then construct the functions $q_{i}\left(x_{0}, \overline{\boldsymbol{e}}_{i}\right)$ as in (123); 4) Calculate the constants $\epsilon_{2}$ and functions $\Psi_{i}\left(x_{0}, \overline{\boldsymbol{e}}_{i}\right)$ as in (76), then construct the functions $p_{i}\left(x_{0}, \overline{\boldsymbol{e}}_{i}\right)$ and $\alpha_{i}\left(x_{0}, \overline{\boldsymbol{e}}_{i}\right)$ as in (125); 5) Calculate the inverse optimal control $u_{1}^{*}$ as in (126). The above steps should be almost the same as for the case $x_{0}\left(t_{0}\right)=0$ as shown in Table I.

\section{CONCLUSIONS}

A design of global asymptotic and optimal stabilizers with respect to a meaningful cost function for stochastic nonholonomic systems has been proposed. Both of the $x_{0^{-}}$and $\boldsymbol{x}$-subsystems are affine in stochastic disturbances. A class of fairly general Lyapunov functions was developed for the control design. By proposing modified Sontag's formula, the control design is less tedious than those proposed for strict feedback systems in [17]. Since the Lyapunov functions are not restricted to quadratic or quartic forms, future work is to utilize the control design techniques in this paper to improve control performance of those in [34], [35], [36] proposed for underactuated surface ships and underwater vehicles by 1) addressing system both state-dependent and state-independent stochastic disturbances, and 2) considering optimality.

\section{ACKNOWLEDGMENTS}

The author would like to express his sincere gratitude to the Editor-in-Chief, the Associate Editor, and the reviewers for their helpful comments.

APPENDIX A

PROOF OF LEMMA 2.2

From (11), we have

$$
d \phi(t)=a(t) \phi(t) d t+\sum_{i=1}^{r} c_{i}(t) \phi(t) d w_{i}
$$

Define $\xi(t)=x\left(t_{0}\right)+\int_{t_{0}}^{t} \frac{1}{\phi(s)} b(s) d s$, which yields $d \xi(t)=\frac{1}{\phi(t)} b(t) d t$. Let $x(t)=\phi(t) \xi(t)$. Applying stochastic differentiation formula (13) gives

$$
d x(t)=d \phi(t) \xi(t)+\phi(t) d \xi(t)+d \phi(t) d \xi(t)=(a(t) x+b(t)) d t+x \sum_{i=1}^{r} c_{i}(t) d w_{i},
$$

which verifies (9).

\section{APPENDIX B}

\section{EXPLICIT DERIVATION OF $u_{0}^{*}$}

We use the Legendre-Fenchel transform to rewrite the optimal control $u_{0}^{*}$ as follows. Multiplying the numerator and denominator of the right hand side of (41) by $\left|R_{0}^{-1 / 2} \gamma_{0}^{\prime} x_{0}^{2 k-1}\right|$ then using the Legendre- 
Fenchel transform (7) and (43), we have

$$
\begin{aligned}
u_{0}^{*} & =-\frac{\beta_{0}}{2} R_{0}^{-1} \gamma_{0}^{\prime} x_{0}^{2 k-1} \frac{\left(\sigma_{0}^{\star}\right)^{-1}\left(\left|R_{0}^{-1 / 2} \gamma_{0}^{\prime} x_{0}^{2 k-1}\right|\right)\left|R_{0}^{-1 / 2} \gamma_{0}^{\prime} x_{0}^{2 k-1}\right|}{\left|R_{0}^{-1 / 2} \gamma_{0}^{\prime} x_{0}^{2 k-1}\right|^{2}} \\
& =-\frac{\beta_{0}}{2} R_{0}^{-1} \gamma_{0}^{\prime} x_{0}^{2 k-1}\left[\frac{\ell \sigma_{0}\left(\left|R_{0}^{-1 / 2} \gamma_{0}^{\prime} x_{0}^{2 k-1}\right|\right)}{\left|R_{0}^{-1 / 2} \gamma_{0}^{\prime} x_{0}^{2 k-1}\right|^{2}}+\frac{\sigma_{0}\left(\left(\sigma_{0}^{\star}\right)^{-1}\left(\left|R_{0}^{-1 / 2} \gamma_{0}^{\prime} x_{0}^{2 k-1}\right|\right)\right)}{\left|R_{0}^{-1 / 2} \gamma_{0}^{\prime} x_{0}^{2 k-1}\right|^{2}}\right] \\
& =-\frac{\beta_{0}}{2} R_{0}^{-1} \gamma_{0}^{\prime} x_{0}^{2 k-1}\left[\frac{\left(k_{0}+\sqrt{c_{0}+p_{0}^{2}\left(x_{0}\right)}\right) x_{0}}{R_{0}^{-1} \gamma_{0}^{\prime} x_{0}^{2 k-1}}+\frac{\sigma_{0}\left(\left(\sigma_{0}^{\star}\right)^{-1}\left(\left|R_{0}^{-1 / 2} \gamma_{0}^{\prime} x_{0}^{2 k-1}\right|\right)\right)}{\left|R_{0}^{-1 / 2} \gamma_{0}^{\prime} x_{0}^{2 k-1}\right|^{2}}\right],
\end{aligned}
$$

which can be written as (45).

\section{APPENDIX C}

PROOF OF THEOREM 3.1

\section{A. Part 1}

With the inverse pre-optimal control $u_{0}^{\diamond}$, we prove in this part existence, uniqueness and asymptotic convergence of the solution $x_{0}(t)$ to zero in probability, and nonzero crossing of the control $u_{0}^{\diamond}$.

1) Case $x_{0}\left(t_{0}\right) \neq 0$ : The inequality (34) implies from Theorem 2.1 that the solution $x_{0}(t)$ of the $x_{0^{-}}$ subsystem exists and is unique, and asymptotically converge to zero in probability. Now substituting the control $u_{0}^{\diamond}$ given by (32) into the first equation of (1) and using (29) give

$$
d x_{0}=-\left(k_{0}+\sqrt{c_{0}+p_{0}^{2}\left(x_{0}\right)}\right) x_{0} d t+x_{0} \varphi_{00}^{T}\left(x_{0}\right) d \boldsymbol{w} .
$$

Since we have already proved that the solution $x_{0}(t)$ of (136) exists and is unique, $p_{0}\left(x_{0}(t)\right)$ and $\varphi_{00}\left(x_{0}(t)\right)$ can be viewed as functions of $t$. This in turn implies that the system (136) can be regarded as a timevarying linear stochastic differential equation, whose solution exists and is unique. Therefore, applying Lemma 2.2 to (136) results in

$$
x_{0}(t)=x_{0}\left(t_{0}\right) \mathrm{e}^{\Omega_{0}(t)},
$$

where

$$
\Omega_{0}(t)=\int_{t_{0}}^{t}\left(-\left(\left(k_{0}+\sqrt{1+p_{0}^{2}\left(x_{0}(s)\right)}+p_{0}\left(x_{0}(s)\right)\right)+\frac{1}{2}\left\|\boldsymbol{\varphi}_{00}\left(x_{0}(s)\right)\right\|^{2}\right) d s+\boldsymbol{\varphi}_{00}^{T}\left(x_{0}(s)\right) d \boldsymbol{w}(s)\right) .
$$

The equation (137) shows that $x_{0}(t)$ does not cross zero. Thus from (32), $u_{0}^{\diamond}(t)$ does not cross zero.

2) Case $x_{0}\left(t_{0}\right)=0$ : The inequality (40) implies from Theorem 2.1 that the solution $x_{0}(t)$ of of the $x_{0}$-subsystem exists and is unique for both cases $t \leq t_{s}^{\diamond}$ and $t>t_{s}^{\diamond}$. We now show that $x_{0}\left(t_{s}^{\diamond}\right)$ is actually non-zero. Substituting the control $u_{0}$ given by (35) into the first equation of (1) and using (29) give

$$
d x_{0}=\left[-\left(k_{0}+\sqrt{c_{0}+p_{0}^{2}\left(x_{0}\right)}\right) x_{0}+\eta_{0}\right] d t+x_{0} \varphi_{00}^{T}\left(x_{0}\right) d \boldsymbol{w}, \quad \text { for } t \leq t_{s}^{\diamond} .
$$

Since we have already proved that $x_{0}(t)$ of (139) exists and is unique, $p_{0}\left(x_{0}(t)\right)$ and $\varphi_{00}\left(x_{0}(t)\right)$ can be considered as functions of $t$, i.e., the system (139) can be viewed as a time-varying linear stochastic differential equation, whose solution exists and is unique. Hence, applying Lemma 2.2 to (139) results in

$$
x_{0}(t)=\left(x_{0}\left(t_{0}\right)+\int_{t_{0}}^{t} \mathrm{e}^{-\Omega_{0}(s)} \eta_{0} d s\right) \mathrm{e}^{\Omega_{0}(t)}=\left(\int_{t_{0}}^{t} \mathrm{e}^{-\Omega_{0}(s)} \eta_{0} d s\right) \mathrm{e}^{\Omega_{0}(t)}, \quad \text { for } t \leq t_{s}^{\diamond},
$$

where we have used the fact that the case $x_{0}\left(t_{0}\right)=0$ is being considered. The equation (140) shows that $x_{0}\left(t_{s}^{\diamond}\right)$ is non-zero. Proof of asymptotic convergence of $x_{0}(t)$ to zero in probability and that non-zero crossing of $u_{0}(t)$ is the same as for the case $x_{0}\left(t_{0}\right) \neq 0$ by viewing $t_{s}^{\diamond}$ as the initial time. 


\section{B. Part 2}

Since $\mathcal{L} V_{0}\left(x_{0}\right)$ satisfies (46) for the case $x_{0}\left(t_{0}\right) \neq 0$ and (49) for the case $x_{0}\left(t_{0}\right)=0$, the term $R_{1}^{-1} \gamma_{n}^{\prime} e_{n}^{2 k-1} \sigma_{1}\left(\left(\sigma_{1}^{\star}\right)^{-1}\left(\left|R_{1}^{-1 / 2} \gamma_{n}^{\prime} e_{n}^{2 k-1}\right|\right)\right) /\left|R_{1}^{-1 / 2} \gamma_{n}^{\prime} e_{n}^{2 k-1}\right|^{2}$ is nonnegative and continuous away from the origin, and $\beta_{0} \geq 2$, proof of existence, uniqueness and asymptotic convergence of the solution $x_{0}(t)$ to zero in probability, and nonzero crossing of the control $u_{0}^{*}$ and its asymptotic convergence to zero in probability follows the proof in Subsection C-A. Optimality follows directly from Theorem 2.2.

\section{APPENDIX D}

\section{PROOF OF THEOREM 5.1}

We use the following Lyapunov function candidate

$$
V\left(x_{0}, \boldsymbol{e}\right)=V_{0}\left(x_{0}\right)+V_{1}(\boldsymbol{e}),
$$

where $V_{0}\left(x_{0}\right)$ and $V_{1}(\boldsymbol{e})$ are given by (26) and (65), respectively, for proof of Theorem 5.1.

\section{A. Case $x_{0}\left(t_{0}\right) \neq 0$ and case $x_{0}\left(t_{0}\right)=0$ when $t>t_{s}^{*}$}

1) Case of inverse optimal control $u_{0}^{*}$, stabilizing control $u_{1}$, and inverse pre-optimal control $u_{1}^{\diamond}$ : The inverse optimal control $u_{0}^{*}$, and inverse pre-optimal control $u_{1}^{\diamond}$ are given in (45) or (48), and (79), respectively. The corresponding infinitesimal generators $\left.\mathcal{L} V_{0}\left(x_{0}\right)\right|_{(45)}$ or $\left.\mathcal{L} V_{0}\left(x_{0}\right)\right|_{(48)}$, and $\left.\mathcal{L} V_{1}(\boldsymbol{e})\right|_{(79)}$ are given by (46) or the second inequality of (49) and (80), respectively. Therefore, the infinitesimal generators $\left.\mathcal{L} V\left(x_{0}, \boldsymbol{e}\right)\right|_{((45) \text { or (48)) }}$ and $\left.\mathcal{L} V\left(x_{0}, \boldsymbol{e}\right)\right|_{((45) \text { or (48)),(79) }}$ satisfy

$$
\begin{aligned}
& \left.\mathcal{L} V\left(x_{0}, \boldsymbol{e}\right)\right|_{((45) \text { or }(48))} \leq-\left(\frac{\beta_{0} k_{0}}{2}-\epsilon_{10}\right) \gamma_{0}^{\prime} x_{0}^{2 k}-\sum_{i=1}^{n} k_{i} \gamma_{i}^{\prime} e_{i}^{2 k} \\
& \left.\mathcal{L} V\left(x_{0}, \boldsymbol{e}\right)\right|_{((45) \text { or }(48)),(79)} \leq-\left(\frac{\beta_{0} k_{0}}{2}-\left(\epsilon_{10}+\epsilon_{20}\right)\right) \gamma_{0}^{\prime} x_{0}^{2 k}-\sum_{i=1}^{n} k_{i} \gamma_{i}^{\prime} e_{i}^{2 k} .
\end{aligned}
$$

Since $k_{0}$ is chosen as in (33), $\epsilon_{10}+\epsilon_{20}$ is strictly less than $2 \epsilon$ and $\beta_{0} \geq 2,\left(\frac{\beta_{0} k_{0}}{2}-\epsilon_{10}\right)$ and $\left(\frac{\beta_{0} k_{0}}{2}-\left(\epsilon_{10}+\epsilon_{20}\right)\right)$ are positive constants. Therefore, Theorem 2.1 implies that the solution $\boldsymbol{x}(t)$ of the $\boldsymbol{x}$-subsystem exists and is unique, and asymptotically converges to zero in probability.

2) Case of inverse optimal controls $u_{0}^{*}$ and $u_{1}^{*}$ : The inverse optimal controls $u_{0}^{*}$ and $u_{1}^{*}$ are given in (45) or (48) and (85), respectively. The corresponding infinitesimal generators $\left.\mathcal{L} V_{0}\left(x_{0}\right)\right|_{(45)}$ or $\left.\mathcal{L} V_{0}\left(x_{0}\right)\right|_{(48)}$ and $\left.\mathcal{L} V_{1}(\boldsymbol{e})\right|_{(85)}$ are given by (46) or the second inequality of (49) and (86), respectively. Therefore, the infinitesimal generator $\mathcal{L} V\left(x_{0}, \boldsymbol{e}\right)_{((45)}$ or (48)),(85) satisfies

$$
\mathcal{L} V\left(x_{0}, \boldsymbol{e}\right)_{((45) \text { or }(48)),(85)} \leq-\left(\frac{\beta_{0} k_{0}}{2}-\left(\epsilon_{10}+\epsilon_{20}\right)\right) \gamma_{0}^{\prime} x_{0}^{2 k}-\left[\sum_{i=1}^{n-1} k_{i} \gamma_{i}^{\prime} e_{i}^{2 k}+\frac{\beta_{1} k_{n}}{2} \gamma_{n}^{\prime} e_{n}^{2 k}\right]
$$

Since $\left(\frac{\beta_{0} k_{0}}{2}-\left(\epsilon_{10}+\epsilon_{20}\right)\right)$ is a positive constant as shown above, Theorem 2.1 implies that the solution $\boldsymbol{x}(t)$ of the $\boldsymbol{x}$-subsystem exists and is unique, and asymptotically converge to zero in probability. Optimality follows directly from Theorem 2.2 with a note that $L_{\boldsymbol{G}_{2}} V(\boldsymbol{e})=\gamma_{n}^{\prime} e_{n}^{2 k-1}$.

\section{B. Case $x_{0}\left(t_{0}\right)=0$ when $t \leq t_{s}^{*}$}

The inverse optimal control $u_{0}^{*}$, inverse pre-optimal control $u_{1}^{\diamond}$, and inverse optimal control $u_{1}^{*}$ are given in (47), (95), and (97), respectively. The corresponding infinitesimal generators $\left.\mathcal{L} V_{0}\left(x_{0}\right)\right|_{(47)},\left.\mathcal{L} V_{1}(\boldsymbol{e})\right|_{(95)}$, and 
$\left.\mathcal{L} V_{1}(\boldsymbol{e})\right|_{(97)}$ are given by the first inequality of (49), (96), and (99), respectively. Therefore, the infinitesimal generators $\left.\mathcal{L} V\left(x_{0}, \boldsymbol{e}\right)\right|_{(47))},\left.\mathcal{L} V\left(x_{0}, \boldsymbol{e}\right)\right|_{(47),(95)}$, and $\mathcal{L} V\left(x_{0}, \boldsymbol{e}\right)_{(47),(97)}$ satisfy

$\left.\mathcal{L} V\left(x_{0}, \boldsymbol{e}\right)\right|_{(47))} \leq-\left(\frac{\beta_{0}}{2} k_{0}-\varepsilon_{1} a_{0}^{\frac{1}{2 k-1}}-\epsilon_{10}\right) \gamma_{0}^{\prime} x_{0}^{2 k}-\sum_{i=1}^{n} k_{i} \gamma_{i}^{\prime} e_{i}^{2 k}+\varepsilon_{2} \eta_{0}^{2 k}+\bar{\Phi}_{0}$,

$\left.\mathcal{L} V\left(x_{0}, \boldsymbol{e}\right)\right|_{(47),(95)} \leq-\left(\frac{\beta_{0}}{2} k_{0}-\varepsilon_{1} a_{0}^{\frac{1}{2 k-1}}-\left(\epsilon_{10}+\epsilon_{20}\right)\right) \gamma_{0}^{\prime} x_{0}^{2 k}-\sum_{i=1}^{n} k_{i} \gamma_{i}^{\prime} e_{i}^{2 k}+\varepsilon_{2} \eta_{0}^{2 k}+\bar{\Phi}_{0}+\bar{\Psi}_{0}$.

$\left.\mathcal{L} V\left(x_{0}, \boldsymbol{e}\right)\right|_{(47),(97)} \leq-\left(\frac{\beta_{0}}{2} k_{0}-\varepsilon_{1} a_{0}^{\frac{1}{2 k-1}}-\left(\epsilon_{10}+\epsilon_{20}\right)\right) \gamma_{0}^{\prime} x_{0}^{2 k}-\left[\sum_{i=1}^{n-1} k_{i} \gamma_{i}^{\prime} e_{i}^{2 k}+\frac{\beta_{1} k_{n}}{2} \gamma_{n}^{\prime} e_{n}^{2 k}\right]+\varepsilon_{2} \eta_{0}^{2 k}+\bar{\Phi}_{0}+\bar{\Psi}_{0}$

Since $k_{0}$ is chosen as in (33), $\epsilon_{10}$ and $\epsilon_{20}$ are positive constants such that $\epsilon_{10}+\epsilon_{20}$ is strictly less than $2 \epsilon$, $\beta_{0} \geq 2$, and $\varepsilon_{1}$ and $\varepsilon_{2}$ are defined in (39), $\left(\frac{\beta_{0}}{2} k_{0}-\varepsilon_{1} a_{0}^{\frac{1}{2 k-1}}-\epsilon_{10}\right)$ and $\left(\frac{\beta_{0}}{2} k_{0}-\varepsilon_{1} a_{0}^{\frac{1}{2 k-1}}-\left(\epsilon_{10}+\epsilon_{20}\right)\right)$ are larger than a positive constant. Therefore, Theorem 2.1 implies that the solution $\boldsymbol{x}(t)$ of the $\boldsymbol{x}$-subsystem exists and is unique.

\section{REFERENCES}

[1] K. D. Do and J. Pan, "Adaptive global stabilization of nonholonomic systems with strong nonlinear drifts," Systems and Control Letters, vol. 46, no. 3, pp. 195-205, 2002.

[2] Z. Qu, R. Hull, and J. Wang, "Globally stabilizing adaptive control design for nonlinearly parameterized systems," IEEE Transactions on Automatic Control, vol. 51, no. 6, pp. 1073-1079, 2006.

[3] T. Fukao, H. Nakagawa, and N. Adachi, "Adaptive tracking control of a nonholonomic mobile robot," IEEE Transactions on Robotics and Automation, vol. 16, no. 5, pp. 609-615, 2000.

[4] Z. Yu and G. S. Chirikjian, "Probabilistic models of dead-reckoning error in nonholonomic mobile robots," Proceedings of the 2003 IEEE International Conference on Robotics \& Automation, vol. 2, pp. 1594-1599, 2003.

[5] R. W. Brockett, "Asymptotic stability and feedback stabilization," in Differential Geometric Control Theory (R. W. Brockett, R. S. Millman, and H. J. Sussmann, eds.), pp. 181-191, Boston: Birkhauser, 1983.

[6] I. Kolmanovsky and N. H. McClamroch, "Developments in nonholonomic control problems," IEEE Control Systems Magazine, no. 6, pp. 20-36, 1995.

[7] A. Astolfi, "Discontinuous control of nonholonomic systems," Systems Control \& Letters, vol. 27, pp. 37-45, 1996.

[8] R. McCloskey and R. Murray, "Exponential stabilization of driftless nonlinear control systems using homogeneous feedback," IEEE Transactions on Automatic Control, vol. 42, no. 5, pp. 614-628, 1997.

[9] Z. P. Jiang, "Robust exponential regulation of nonholonomic systems with uncertainties," Automatica, vol. 36, no. 2, pp. 189-209, 2000.

[10] Z. Sun, S. S. Ge, W. Huo, and T. H. Lee, "Stabilization of nonholonomic chained systems via nonregular feedback linearization," Systems and Control Letters, vol. 44, no. 4, pp. 279-289, 2001.

[11] S. S. Ge, Z. Wang, and T. H. Lee, "Adaptive stabilization of uncertain nonholonomic systems by state and output feedback," Automatica, vol. 39, pp. 1451-1460, 2003.

[12] R. Murray and S. Sastry, "Nonholonomic motion planning: Steering using sinusoids," IEEE Transactions on Automatic Control, vol. 38, no. 5, pp. 700-716, 1993.

[13] C. de Wit Canudas, B. Siciliano, and G. Bastin, Theory of Robot Control. London: Springer, 1996.

[14] R. Colbaugh and K. Glass, "Learning control for nonholonomic mechanical systems," NOLCOS'98, pp. 771-776, 1998.

[15] Z. P. Jiang, "Iterative design of time-varying stabilizers for multi-input systems in chained form," Systems and Control Letters, vol. 28, no. 5, pp. 255-262, 1996.

[16] H. J. Kushner, Stochastic Stability and Control. New York: Academic Press, 1967.

[17] M. Krstic and H. Deng, Stabilization of Nonlinear Uncertain Systems. London: Springer, 1998.

[18] R. Khasminskii, Stochastic Stability of Differential Equations. Rockville, Maryland: S \& N International Publisher, 1980.

[19] Y. Zhao, J. B. Yu, and Y. Q. Wu, "State-feedback stabilization for a class of more general high order stochastic nonholonomic systems," International Journal of Adaptive Control and Signal Processing, vol. 25, pp. 687-706, 2011.

[20] F. Gao and F. Yuan, "Adaptive stabilization of stochastic nonholonomic systems with nonlinear parameterization," Applied Mathematics and Computation, vol. 219, no. 16, pp. 8676-8686, 2013.

[21] W. Lin, R. Pongvuthithum, and C. Qian, "Control of high order nonholonomic systems in power chained form using discontinuous feedback," IEEE Transactions on Automatic Control 2002, vol. 47, no. 1, pp. 108-115, 2002.

[22] J. Wang, H. Q. Gao, and H. Li, "Adaptive robust control of nonholonomic systems with stochastic disturbances," Science in China: Series F Information Sciences, vol. 49, no. 2, pp. 189-207, 2006.

[23] W. Feng, Q. Sun, Z. Cao, D. Zhang, and H. Chen, "Adaptive state-feedback stabilization for stochastic nonholonomic mobile robots with unknown parameters," Discrete Dynamics in Nature and Society, vol. 2013, pp. 1-9, 2013.

[24] M. Krstic and Z. H. Li, "Inverse optimal design of input-to-state stabilizing nonlinear controllers," IEEE Transactions on Automatic Control, vol. 43, no. 3, pp. 336-350, 1998.

[25] G. Hardy, J. E. Littlewood, and G. Polya, Inequalities. Cambridge: Cambridge University Press, 2 nd ed., 1989.

[26] I. Karatzas and S. E. Shreve, Brownian motion and stochastic caculus. New York: Springer, 2nd ed., 1991.

[27] K. D. Do, Z. P. Jiang, and J. Pan, "Underactuated ship global tracking under relaxed conditions," IEEE Transactions on Automatic Control, vol. 47, no. 9, pp. 1529-1536, 2002. 
[28] K. D. Do, Z. Jiang, and J. Pan, "On global tracking control of a VTOL aircraft without velocity measurements," IEEE Transactions on Automatic Control, vol. 48, no. 12, pp. 2212-2217, 2003.

[29] K. D. Do, "Formation tracking control of unicycle-type mobile robots with limited sensing ranges," IEEE Transactions on Control Systems Technology, vol. 16, no. 3, pp. 527-538, 2008.

[30] H. Deng and M. Krstic, "Stochastic nonlinear stabilization-Part I: A backstepping design," Systems and Control Letters, vol. 32, no. 3, pp. 143-150, 1997.

[31] H. Deng and M. Krstic, "Stochastic nonlinear stabilization-Part II: Inverse optimality," Systems and Control Letters, vol. 32, no. 3, pp. 151-159, 1997.

[32] E. D. Sontag, "Smooth stabilization implies comprime factorization," IEEE Transactions on Automatic Control, vol. 34, no. 4, pp. 435443, 1989.

[33] R. Sepulchre, M. Jankovic, and P. Kokotovic, Constructive Nonlinear Control. New York: Springer, 1997.

[34] K. D. Do, Z. P. Jiang, and J. Pan, "Universal controllers for stabilization and tracking of underactuated ships," Systems and Control Letters, vol. 47, pp. 299-317, 2002.

[35] K. Do, "Formation control of underactuated ships with elliptical shape approximation and limited communication ranges," Automatica, vol. 48, no. 7, pp. 1380-1388, 2012.

[36] K. D. Do, Z. P. Jiang, and J. Pan, "Robust and adaptive path following for underactuated autonomous underwater vehicle," Ocean Engineering, vol. 31, no. 16, pp. 1967-1997, 2004. 